\title{
DESIGN OF THE SEALEVEL SUSPENDED SOLIDS \\ CONCENTRATION MONITORING SYSTEM
}

\author{
Presented to: \\ DOE National Energy Technology Laboratory \\ 3610 Collins Ferry Road \\ Morgantown, WV 26505
}

Prepared by:

Science and Engineering Associates, Inc.

6100 Uptown Blvd., NE, Suite 700

Albuquerque, NM 87110

(505) $884-2300$

Under Contract DE-AC-21-96-MC33126

September 17, 2001 


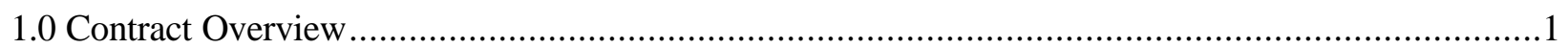

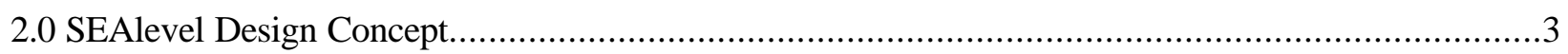

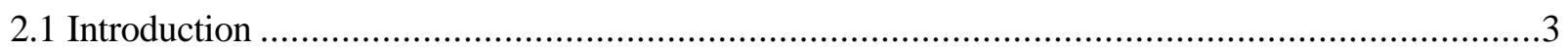

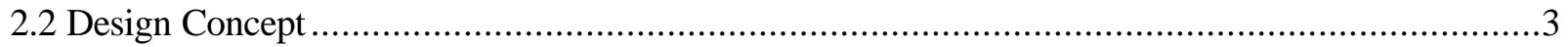

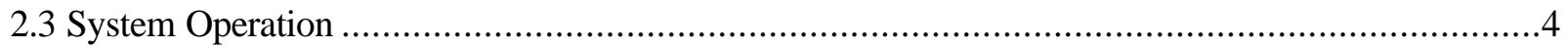

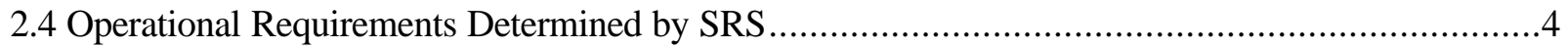

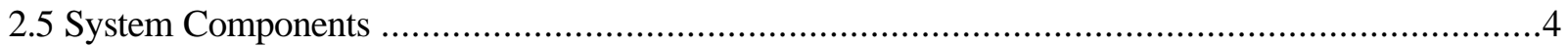

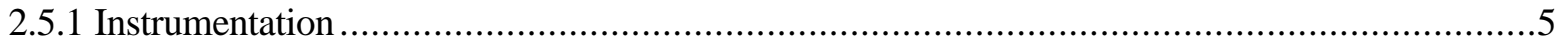

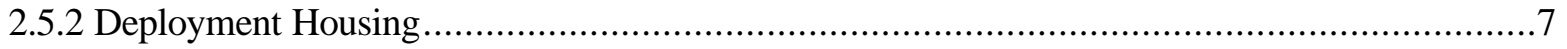

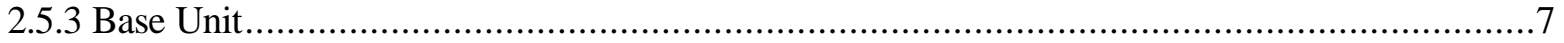

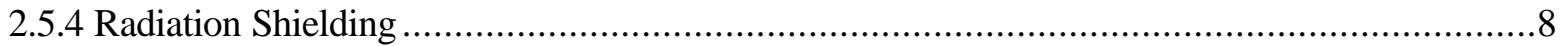

3.0 Comparison of Monitek TSS Sensor with SRS Sensor .......................................................

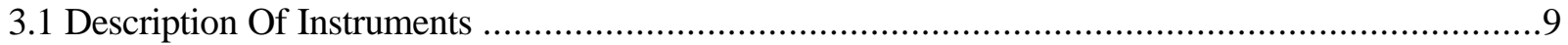

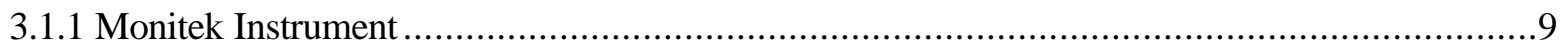

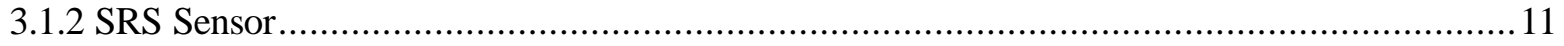

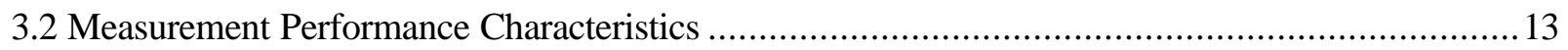

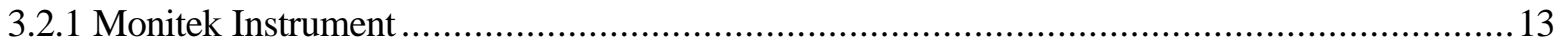

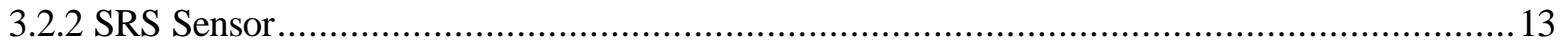

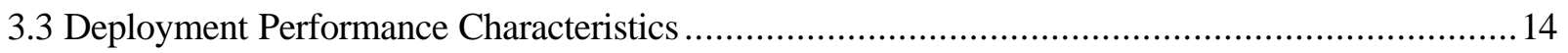

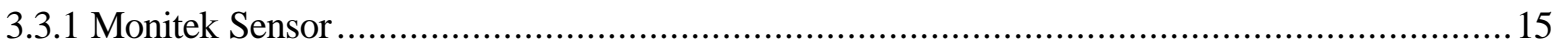

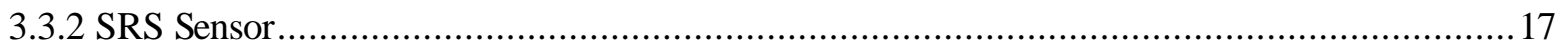

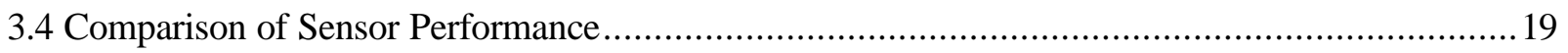

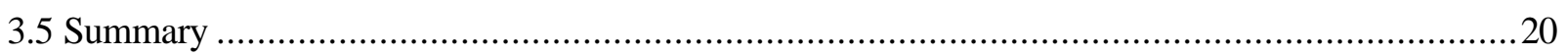

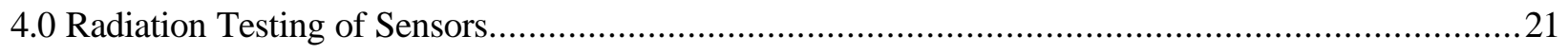

Appendix A - RADIATION TEST PLAN FOR TOTAL SUSPENDED SOLIDS INSTRUMENTS.......22

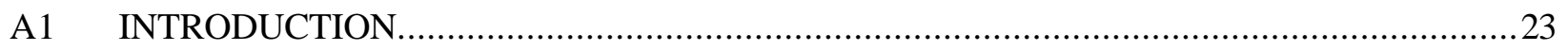

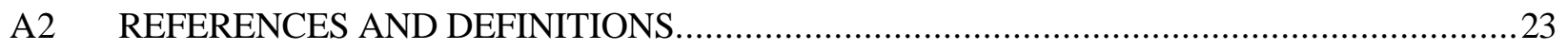

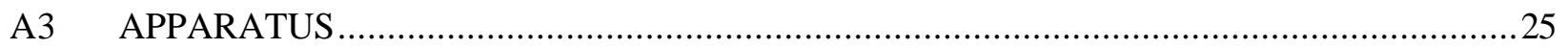

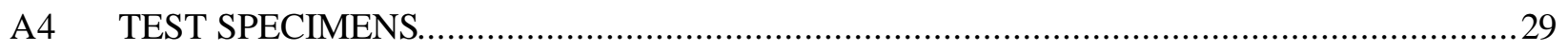

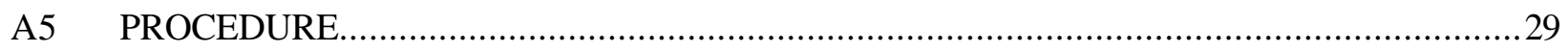

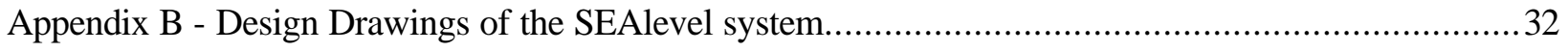




\section{DESIGN OF THE SEALEVEL SUSPENDED SOLIDS CONCENTRATION MONITORING SYSTEM}

\subsection{CONTRACT OVERVIEW}

The SEAlevel project between Science \& Engineering Associates, Inc. (SEA) and the National Energy Technology Laboratory (NETL) in Morgantown, WV (contract DE-AC21-96MC33126) began in direct response to a need expressed by personnel involved with monitoring fluid levels in underground storage tanks at the Hanford Tank Farms. Hanford expressed a desire for an automated monitoring system that could be installed into tanks through liquid observation wells (LOWs). The LOWs are pipes that run from the surface above the tank to the bottom of the tank. The end of the LOW pipe in the tank waste is sealed. Therefore, the LOW provides a clean conduit through which sensors and monitors can be lowered into the tanks. When the SEAlevel project first began, it was understood that the LOWs would be of steel construction, because several existing LOWs at the time were steel pipes and the plans for all future LOW installations were to be with steel pipes. Based on this assumption the SEAlevel monitoring system was to be made using an array of acoustic sensors. However, during the course of the project it was learned that many existing LOWs are of fiberglass construction and that it was the desire of the Tanks Surveillance Group at Hanford to change plans so that all future LOW installations be of fiberglass construction. The Tanks Surveillance Group wanted fiberglass LOWs to allow for a wider range of sensors to be used in the pipes (i.e., sensors that use electromagnetic signals). In response to this, SEA pursued the development of other types of sensors to install in the LOWs in addition to the acoustic sensors that were being developed. It was determined that a capacitance type sensor was well suited for non-metallic LOW pipes. The results of the analysis, testing and design efforts that were undertaken to address the Hanford needs were detailed in a report submitted to the NETL on August 15, 1997, titled, The Sealevel Approach For Monitoring

\section{Liquid Levels In DOE Waste Storage and Processing Tanks.}

The primary focus for application of the SEAlevel monitoring system was initially the tank farms at the Hanford site. However, during the course of the project, the OST Tanks Focus Area identified several other applications of the system at the Savannah River Site. These specifically were to monitor aqueous/organic interfaces and glass frit/water interfaces in waste processing tanks associated with the In-Tank Precipitation (ITP) process at SRS. Laboratory testing of the system in Phase I showed that the accuracy of the approach for the application at Hanford was limited, but was exceptionally well suited for the waste tank applications at SRS. Therefore, the target application site for the technology was changed from Hanford to SRS. As such, the SEAlevel project was redirected to address this need.

Phase II of the SEAlevel program was to address fabrication and testing of a prototype capacitance detector array to support monitoring of the aqueous-organic interface level in the Defense Waste Processing Facility (DWPF) tanks. The aqueous-organic interface measurement was in direct support of the In-Tank Precipitation (ITP) process. However, the ITP process was suspended pending an evaluation of alternative treatment methodologies. This action, undertaken by the DOE Savannah River Site, put into question the need for the capacitance array sensor. As

a result of this change, SEA was requested to undertake a change in the scope for Phase II of this 
contract in order to develop a system for monitoring the vertical profile of total suspended solids (TSS) in waste processing tanks supporting the DWPF. Through the course of the project, multiple technical interchange meetings and design reviews were held with the TFA and the Savannah River Site. This included numerous technical conference calls in addition to the formal meetings listed below;

- Technical specifications obtained through meeting with end users at SRS - March 26, 1998

- Project status presented at CMST Annual Review - April 8, 1998

- Preliminary design submitted to SRS - October 1998

- Design review meeting at SRS - December 1998

- Design review meeting at SRS - April 1999

This report documents the efforts and results of the portion of the project directed toward solving the stated need at the SRS. Section 2 provides an overview of the design concept and its technical basis. The actual design drawings for the system are included as an attachment to this report under Appendix B. The core component of the tank monitoring system is the suspended solids instrumentation. As part of the effort, multiple systems were evaluated and two candidate sensor systems were selected. Section 3 provides an overview of the merits of these two systems. A key component of the evaluation of the sensor systems was the ability of the sensor to withstand high radiation doses. Testing of the two systems was carried out at a high dose Co-60 test facility. The test plan that was used to conduct those tests is included in Appendix A 


\subsection{SEALEVEL DESIGN CONCEPT}

\subsection{Introduction}

Science \& Engineering Associates, Inc. (SEA) designed a system for monitoring suspended solids concentration in waste processing tanks at the DOE Savannah River Site. The goal of this project was to design and build a system for deploying a suspended solids concentration sensor into SRS tanks being used to prepare waste for the Defense Waste Processing Facility. Initial deployment of the system in a waste tank was scheduled to occur in fiscal year 2000, but was never fielded.

A critical aspect of the SEAlevel design process was to seek out critical reviews from appropriate personnel at the SRS, such as management, operations, and safety personnel. A preliminary design was submitted for review by such SRS personnel. As part of that process, a design review meeting was held at the SRS. Comments from the preliminary design review were incorporated into the formal design presented here. The system design was presented at a final design review meeting held in April, 1999 at the SRS. Fabrication of the SEAlevel system was to begin immediately after the design review meeting. However, it was determined at that time that the technical need expressed by SRS did not exist at the operational le vel. As such, the system was never fabricated.

\subsection{Design Concept}

The goal of the SEAlevel design was to provide a deployment package for lowering a suspended solids concentration sensor into waste processing tanks at SRS. A schematic of the design is shown in Figure 1.

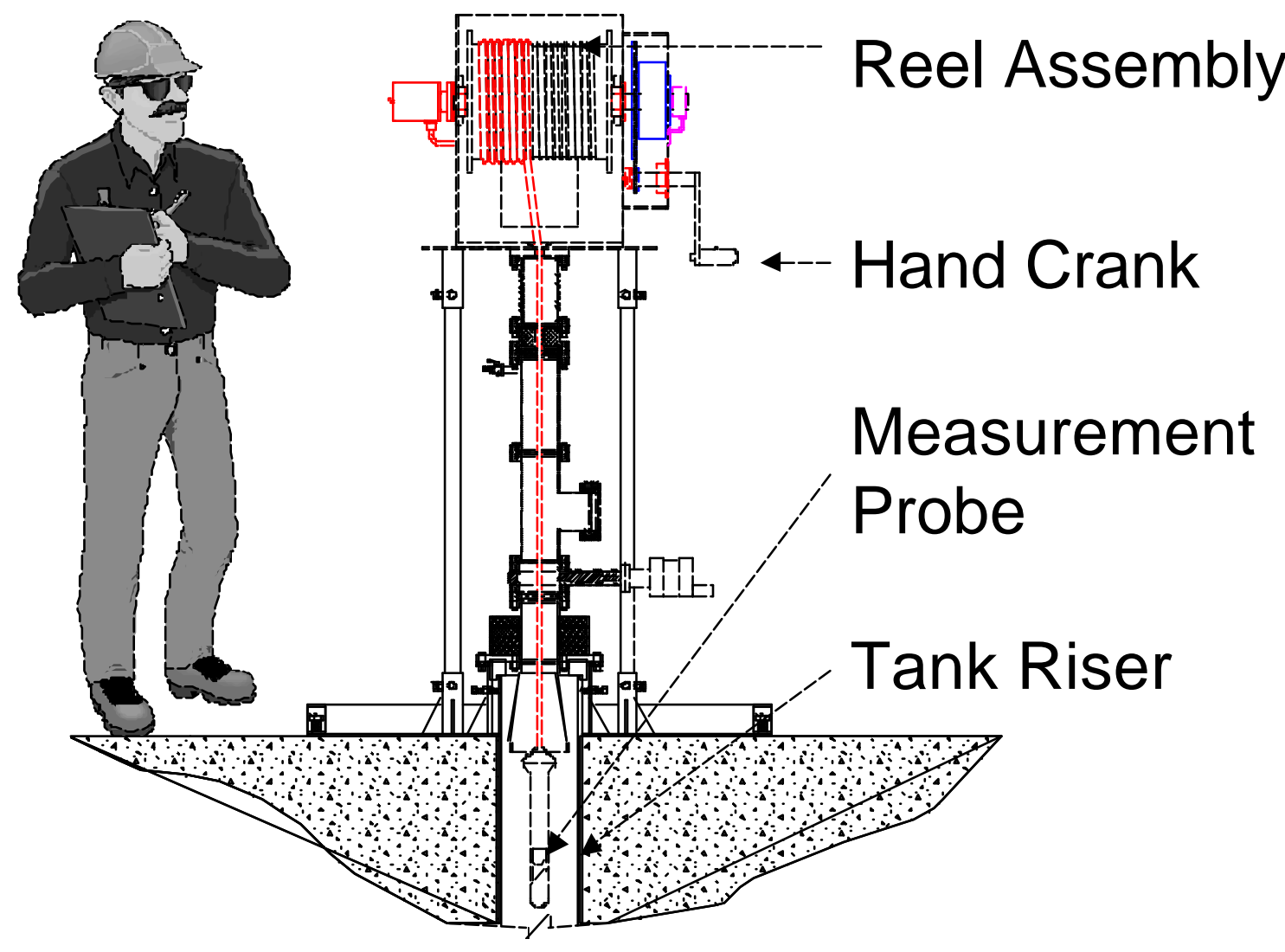

Figure 1. Schematic representation of the SEAlevel system. 


\subsection{System Operation}

In order to understand the functional features of the deployment system design, the basic operation of the SEAlevel system is described here. The primary objective of the system is to determine the elevation within the tank where the suspended solids concentration level is greater than 0.1 percent by weight. To determine this, a measurement probe will be lowered into the tank waste. Periodic measurements of the instrument output will be taken at predetermined intervals (for instance every 3 inches). An operator will manually record both the suspended solids concentration data and the elevation at which the measurement is taken. A data entry form will be prepared to use in conjunction with the system. The measurements will be repeated until the instrument readings show that the probe is below the liquid elevation where the suspended solids concentration is greater than $0.1 \mathrm{wt}$. \%. Additional measurements can be taken at lower elevations to determine the suspended solids concentration profile with depth. The upper limit for these measurements will be at least $1.0 \mathrm{wt}$. \%.

Upon retrieval, spray ring jets will be activated at two elevations to clean the probe cabling as it is pulled out of the tank waste. The lower spray ring will also clean the probe itself once it is fully retrieved to its home position. The supply for the cleaning system will be from the tank-farm inhibited water supply. With the probe in the home position a gate valve will be shut to prevent the sensor from being inadvertently lowered into the tank and to allow for occasional introduction of a known suspended solids solution for in-situ probe calibrations. Both the inhibited water used for the cleaning system and the calibration fluid will be discharged into the waste tank.

It was envisioned that suspended solids concentration profile data would be obtained in this manner on a daily basis during each settle and decant phase of a sludge processing cycle. In-situ calibrations were likely to take place once during each settle and decant phase of the waste processing.

\subsection{Operational Requirements Determined by SRS}

The operating requirements of the system as determined through design meetings with SRS were to be as follows;

- 2 year operating lifetime of the probe

- $1.26 \mathrm{Mrad}(\mathrm{Si})$ total dose requirement of the probe, which was derived using the assumption that the probe would be in the waste a maximum of 2 hours per day over the two year lifetime ${ }^{1}$.

- $0.1 \mathrm{wt} . \%$ to $1.0 \mathrm{wt}$ \% minimum measurement range

- Ambient conditions (upper limits)

$$
\begin{array}{ll}
- & 1000 \mathrm{R} / \mathrm{hr} \\
- & \mathrm{pH} 13.9 \\
- & 60^{\circ} \mathrm{C}
\end{array}
$$

\subsection{System Components}

The deployment system that will lower the suspended solids concentration probe into the tank waste can be considered in three functional units; 1) the system instrumentation 2) the deployment housing and 3) the base unit. The instrumentation consists of the measurement probe, the depth measurement instrumentation, a temperature probe, and the instrumentation for displaying all of the data obtained with the system. The deployment housing contains all of the

\footnotetext{
${ }^{1}$ Conversion factor from $\mathrm{R} / \mathrm{hr}$ to $\mathrm{rad}(\mathrm{Si}) / \mathrm{hr}$ is 0.865
} 
mechanisms for deploying \& retrieving, cleaning, and calibrating the suspended solids measurement probe. The base unit serves as the interface between the tank riser and the deployment housing. Each of these three aspects of the design is discussed individually.

\subsubsection{Instrumentation}

The SEAlevel instrumentation includes all portions of the system related to measurement of suspended solids concentration profiles. The primary components are the measurement probe that is lowered into the tank waste and the transmitter unit connected to the probe that is located at the surface above the waste tank. The measurement probe is a model CSK suspended solids measurement probe manufactured by Monitek Technologies, Inc. of Dusseldorf Germany. The probe is manufactured with a stainless steel housing and sapphire windows for the optically based sensor. All gasket material used in the probe is Ethylene Propylene Diene Terpolymer (EPDM), which is both radiation and chemically resistant. For instance, radiation damage data indicates that EPDM exhibits no observable radiation damage effects at doses up to approximately $8 \mathrm{Mrad}$ and only incurs incipient to mild damage up to nearly $100 \mathrm{Mrad}$. Furthermore, EPDM is listed as being unaffected or only slightly affected in 50\% aqueous caustic soda solutions. Since the probe will be lowered into a hydrogen gas environment, the probe will be procured as an intrinsically safe instrument.

The measurement probe is connected to a transmitter that is located at the surface above the tank. The transmitter unit serves as the power supply and instrument readout for the probe. Output from the encoder will be displayed with a digital readout mounted next to the transmitter. The probe will be connected to the transmitter via a 50-foot cable that is wound onto a reel assembly. The cable at the reel assembly will be coupled to the transmitter through a slip ring assembly. The specifications of the Monitek probe and its associated transmitter are shown in Table 1.

The depth of the probe into a tank will be measured with an optical rotary encoder mounted to the shaft of the probe deployment reel. An RTD incorporated into the probe housing will allow for measurement of the tank temperature.

Table 1. Specifications of the Monitek suspended solids probe

\begin{tabular}{|l|l|}
\hline \multicolumn{2}{|c|}{ CSK PROBE } \\
\hline Material & $316 \mathrm{SS}$ \\
\hline Measurement range & $0-0.1 \ldots 10 \%$ \\
\hline Gaskets & EPDM \\
\hline Temperature & $60^{\circ} \mathrm{C}$ \\
\hline Pressure & 3 bar \\
\hline Detector system & IR \\
\hline Measurement gap & $5 \mathrm{~mm}$ \\
& \\
\hline Weight & $\begin{array}{l}\text { Approximately 2 } \\
\text { (without shield housing) }\end{array}$ \\
\hline
\end{tabular}




\begin{tabular}{|l|l|}
\hline \multicolumn{2}{|c|}{ TRANSMITTER } \\
\hline Measurement units & $\begin{array}{l}\text { G/l, ppm, mg/l, TE/F, FTU, } \\
\text { EBC, wt.\%, Color - AU }\end{array}$ \\
\hline Zero point/ final value & Free programmable \\
\hline Display & Alpha-numerical \\
\hline Output & $4-20 \mathrm{~mA}$ \\
\hline Temperature & $-30^{\circ} \mathrm{C} . .50^{\circ} \mathrm{C}$ \\
\hline
\end{tabular}

Examples of the performance of the suspended solids measurement system are shown in Figure 2. The direct output of the sensor is plotted as a function of the known wt. \% concentration of Kaolinite solutions.

\section{Plot of Signal Output vs. Wt.\% Kaolinite}

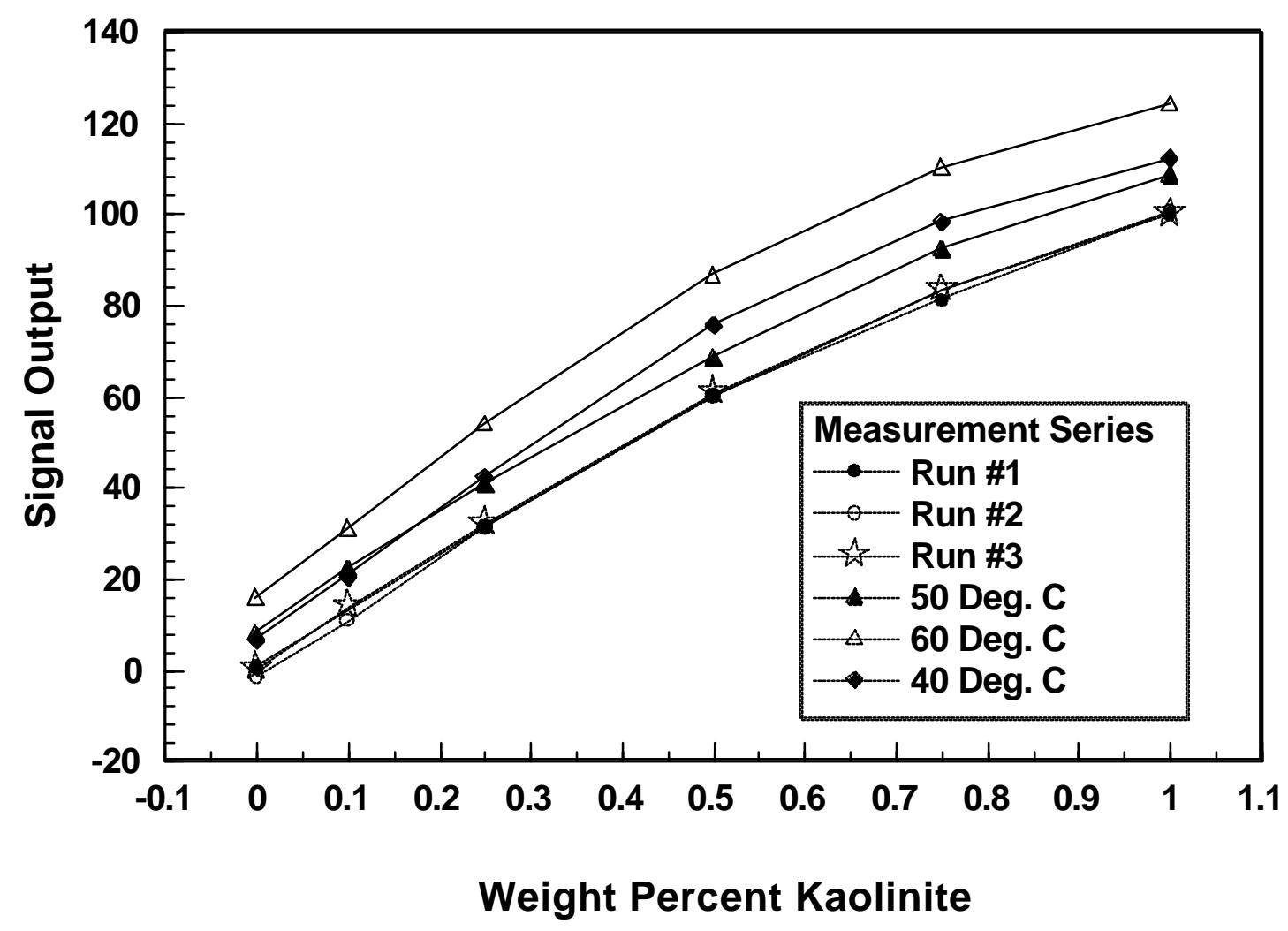

Note: Runs 1-3 were conducted at 20 Deg. C.

Figure 2. Performance of the suspended solids instrumentation. 


\subsubsection{Deployment Housing}

The primary component of the deployment housing is the reel assembly, where the probe cabling is spooled onto the reel and guided by a spiral slot to assure orderly and reproducible spooling of the cable. A pinch roller is incorporated into the housing to prevent the cable from skipping grooves. A hand crank connected to the reel assembly with chain drives controls deployment of the probe. Depth measurements are obtained through an optical rotary encoder attached to the reel assembly shaft. The cable is spooled in a single layer on the reel, which allows for depth into the tank to be measured as a function of reel rotation.

The reel assembly has a failsafe brake connected to the reel shaft to prevent uncontrolled lowering of the probe into the tank and to ensure stationary positioning of the probe during measurements. Release of the brake is controlled through a finger button located on the handle of the crank. The reel can only be moved if an operator depresses the button, which necessarily requires a grip on the crank handle. If the operator losses grip on the handle during deployment or retrieval, or if power is lost, the brake release is deactivated and the reel assembly is locked in place.

Access ports are incorporated into the housing to allow for access to the reel if necessary. A spray nozzle is located at the top of the deployment reel housing to wash down the reel assembly as necessary.

The chain drive and reel brake are enclosed in a compartment of the deployment housing that is isolated from the reel assembly. Thus, access to both can be obtained without exposing workers to the system components that have been in contact with the tank waste.

Directly below the reel assembly housing is a section that provides shielding for the direct shine of the radiation from the tank waste through the riser. Immediately below the shielding section is a small diameter spray ring assembly that provides a final cleaning of the measurement probe cabling prior to spooling on the reel assembly. A site glass is located on the pipe section below the spray ring section to provide a window for taking radiation measurements as the probe is retrieved and for visual inspection of the probe and cabling. A gate valve located below the site glass and attached pipe section serves to seal the deployment unit piping so that calibration fluids can be added to the system.

\section{Design Features}

- Simple, mechanically reliable deployment mechanics

- Failsafe method for keeping probe from falling into tank

- Isolation of most moving parts from the contaminated components

- Design allows for in-situ calibrations

- Decontamination of cable and probe with each retrieval

- Shielding to attenuate radiation shine

\subsubsection{Base Unit}

The base unit serves as the interface between the tank riser and the SEAlevel deployment system. An important aspect of this interface is the configuration of the tank riser, where the riser configuration is assumed to be that shown in SRS drawing B054-2000-008 (as directed by SRS during design review). The riser access is assumed to be an 8-inch pipe coupled to a 10-inch 
flanged pipe that comprises the top 10 inches of the riser pipe. The riser is sealed at two points with the base unit. First, a gasket seal is made between a 13-inch flanged pipe cover on the base unit and the flanged top of the riser pipe. Second, a gasket seal is made at the end of an 8-inch pipe on the base unit that inserts into the riser pipe. This second gasket seal is made between the 8 -inch insert and the transition from 8-inch to 10-inch pipe in the riser. These dual gasket seals will prevent moisture and gases from escaping from the tank riser.

The base unit also serves as the support stand for the deployment reel and support pipe, where four adjustable legs will provide the support. The legs are also removable and the system can be temporarily self-supporting to expedite installation and removal of the SEAlevel system from the tank riser. The footprint of the stand is essentially 66x66 inches and is designed to fit within the diameter of the riser as shown in SRS drawing B054-2000-008.

\section{Design features of the base unit}

- Lead shielding restricts radiation shine

- Gaskets on riser cover and riser insert provide a gas seal between the riser and the environment

- Base plate provides stand for deployment housing so that none of the deployment housing weight is supported by the tank riser

\subsubsection{Radiation Shielding}

Lead shielding is incorporated into the deployment system at various points to eliminate direct radiation shine from the tank waste beyond the physical boundaries of the deployment system. These locations are referred to as the base, secondary, and upper Pb-shields on drawing B0542000-001. An analysis was completed that shows that personnel working in direct contact with any exterior portion of the deployment system will have, at a minimum, 5 inches of lead shielding between them and the direct shine from the tank waste. The only exception to this is the top of the deployment system. However, the height of the system coupled with attenuation through the components of the system may allow for this area to remain unshielded provided procedural restrictions are in place that preclude access to the top of the deployment housing. Alternatively, lead shielding could be incorporated if deemed necessary. 


\subsection{COMPARISON OF MONITEK TSS SENSOR WITH SRS SENSOR}

\subsection{Description Of Instruments}

During the final design review of the system at SRS, in April 1999, it was determined that SRS tank operators used a straightforward approach to measuring the suspended solids interface level with satisfactory results. In essence, the operators constructed a simple photosensor that was lowered into the tank. Deployment of the sensor was achieved by lowering the device into the tank by hand, while an operator watched the probe being lowered into the tank. No extensive shielding measures were required as determined during SEAlevel design reviews. Furthermore, no extensive cleaning requirements were needed as the sensor was retrieved fro the tank. After learning of the existing measurement approach used by SRS tank operators, SEA was asked to do a comparison of the performance of the SRS sensor and the sensor to be used with the SEAlevel system. This section describes the results of that comparison.

\subsubsection{Monitek Instrument}

The Monitek Instrument is a full-featured total suspended solids measurement system designed for industrial process monitoring. It is comprised of two principal components. These are the sensor, and the transmitter unit. The sensor unit houses the light source and detector, and a minimal amount of circuitry necessary to condition the detector signal for transmission to the transmitter unit. The transmitter unit houses the bulk of the instrument electronics in a NEMA enclosure. Figure 3 shows a photo of the Transmitter Unit.

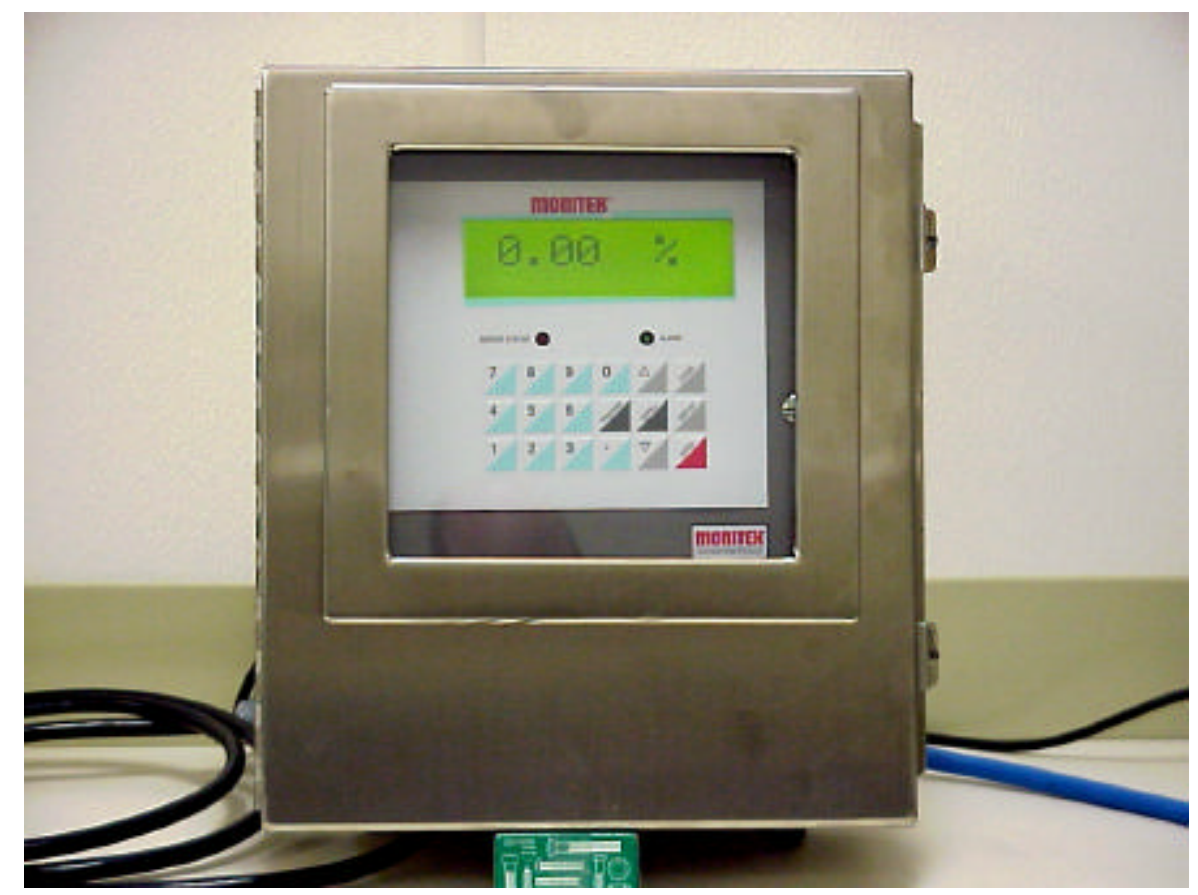

Figure 3. Photo of the Monitek Transmitter Unit housed in a NEMA enclosure. 
Figure 4 shows a photo of the sensor unit. The sensor housing is fabricated from stainless steel with sapphire windows for the light source and photodetector. The light source is an infrared light emitting diode (LED) operating at $880 \mathrm{~nm}$. The photo detector is a silicon photodiode. The Monitek instrument uses the method of optical transmission to measure suspended solids in a liquid medium. The sensor is configured with an optical path length of $5 \mathrm{~mm}$, i.e. a gap of $5 \mathrm{~mm}$ that allows the measurement liquid to flow between the light source and the photo detector. Figure 5 shows a detail of this optical path. With clear liquid in the optical gap, the photodetector measures the full intensity of the light source, giving rise to a maximum photodetector signal. As the concentration of suspended solids increases, light is absorbed and scattered out of the optical path, resulting in a reduction of the photodetector signal. A non-linear calibration curve is used to relate the photodetector signal to the suspended solids concentration. Any number of standards between two and 255 may be used to define this calibration relationship.

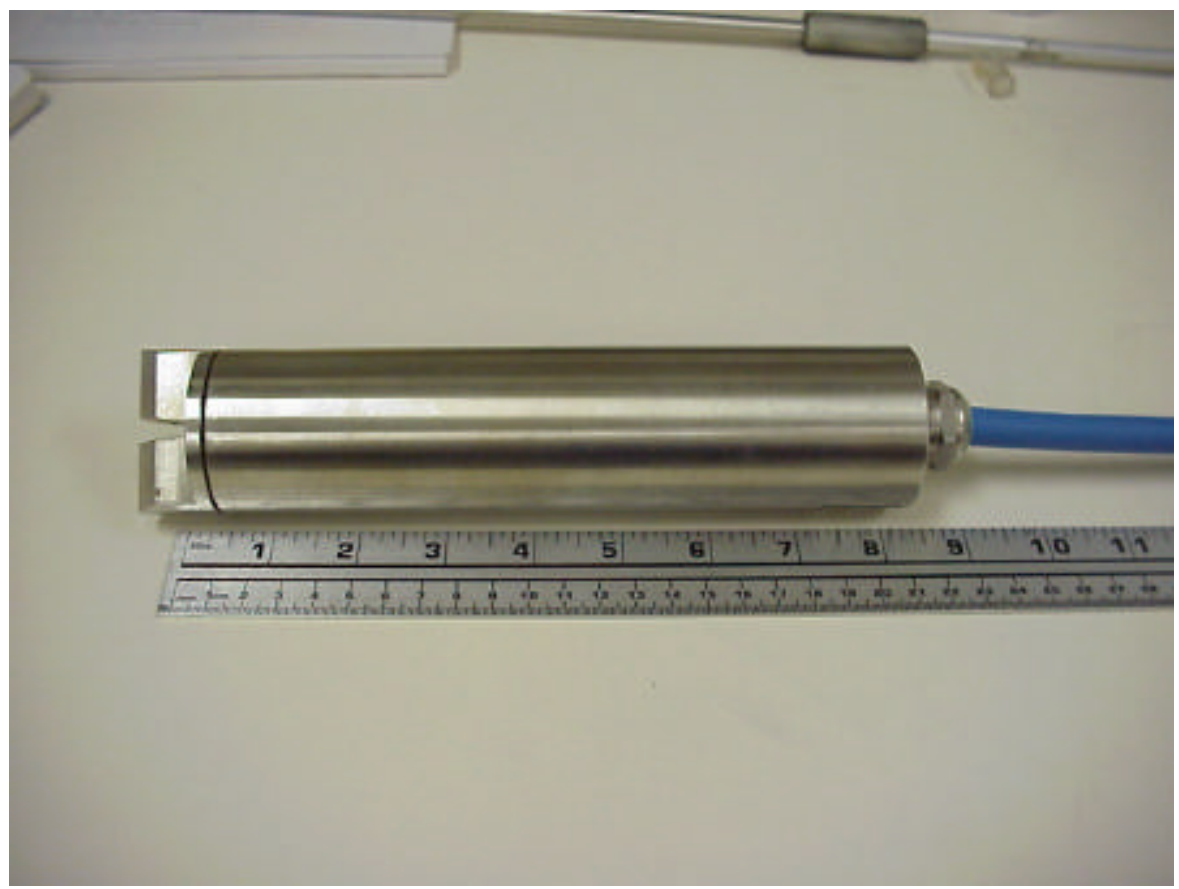

Figure 4. Photo showing the Monitek Sensor. 


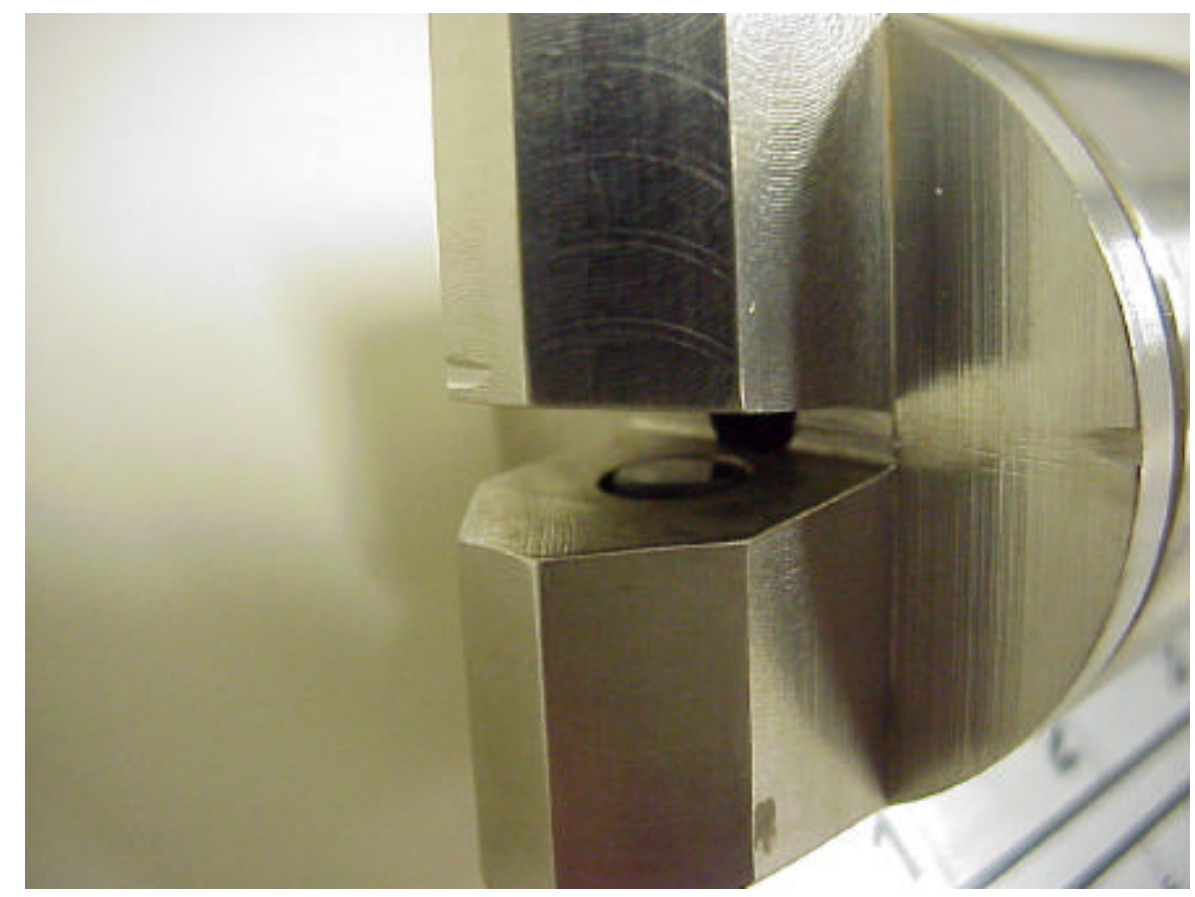

Figure 5. Photo showing details of the optical path. The $5 \mathrm{~mm}$ optical gap is shown, along with one of the sapphire windows.

\subsubsection{SRS Sensor}

The sensor currently in use at the Savannah River Site (SRS) is shown in Figure 6. This sensor is fabricated by SRS from a 6V incandescent bulb and a cadmium sulfide photoresistor packaged in a length of clear vinyl tubing. The vinyl tubing is formed into a loop and held in position with a short length of stiff copper wire. The loop positions the photoresistor in close proximity to the light bulb, leaving a small gap between the two. Figure 7 shows a detail of this gap.

A four-conductor cable is used to extend the connections from the light bulb and the photo resistor a distance of approximately $50 \mathrm{ft}$., to allow the sensor to be lowered into the waste tanks. A $50 \mathrm{ft}$. steel tape is attached to the cable to facilitate depth measurement. The lamp is powered by a 6-Volt battery, and the photoresistance is measured by a multimeter, or ohm meter. A steel weight is attached to the bottom of the vinyltubing loop with electrical tape to provide sufficient weight to sink the sensor through the test liquid. 


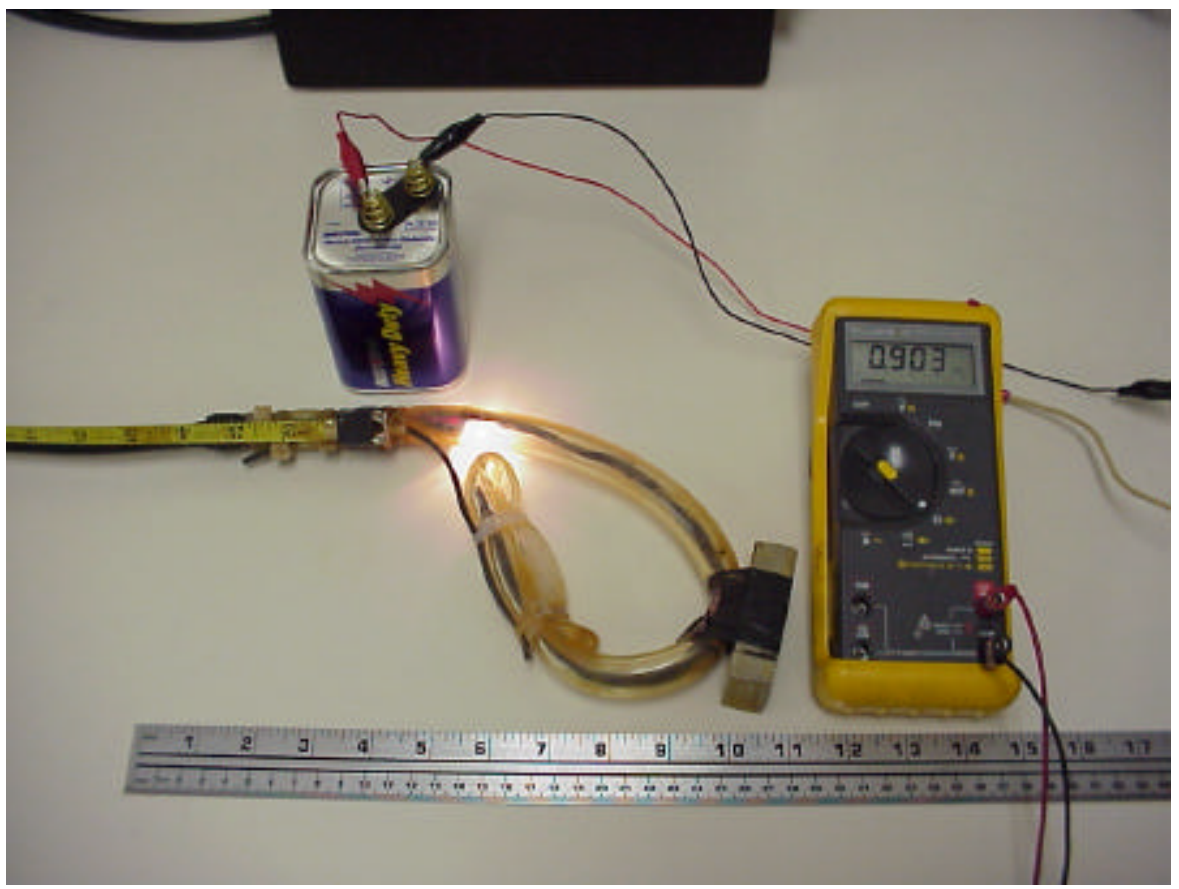

Figure 6. Photo of the SRS sensor currently in use.

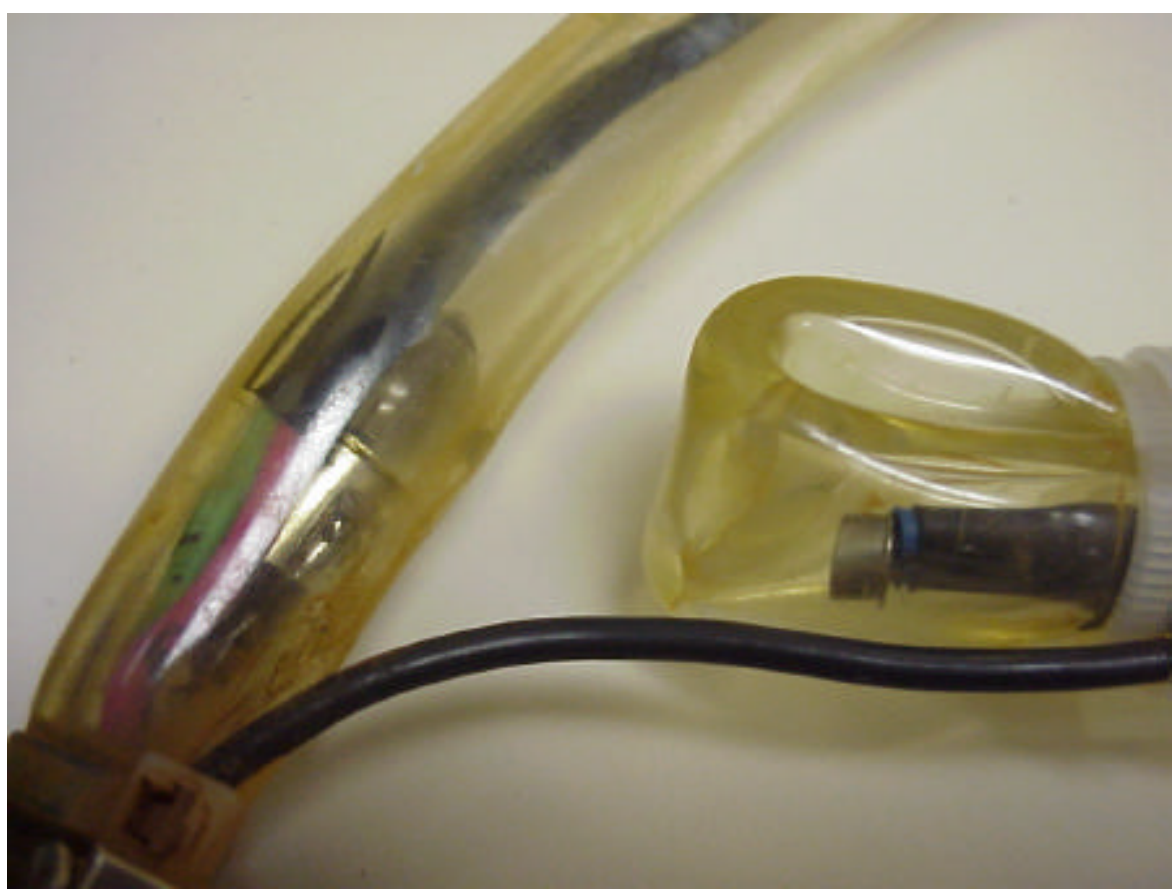

Figure 7. Photo showing details of the arrangement of the lamp and the photoresistor. 


\subsection{Measurement Performance Characteristics}

\subsubsection{Monitek Instrument}

For these measurements the Monitek Instrument was calibrated using a series of six calibration standards The wt.\% solids for these standards are: $0.05,0.1,0.25,0.50,0.75, \& 1.00$ wt.\% solids. These standards were prepared by gravimetrically determining the wt.\% solids of a surrogate provided by SRS, and diluting this appropriately to achieve the target value. The calibration was carried out using the procedures described in the Monitek Operating Instructions Manual. Following the measurements, the standards were measured as unknowns with the instrument. The results of these measurements are shown in Figure 8 along with a best fit linear regression line.

\subsubsection{SRS Sensor}

The SRS sensor was calibrated using the same six standard suspensions used to calibrate the Monitek instrument. The results of these measurements are shown graphically in Figure 9. The relationship between the wt.\% total suspended solids and the measured resistance of the photoresistor is approximately logarithmic. Correspondingly, the plot is shown with the natural $\log$ of the photoresistance plotted, and the regression fitted to the log transformed data. It should be noted that the measurement result obtained in the $1.00 \mathrm{wt} \%$ calibration standard was greater than could be measured with the ohm meter used, and was probably the maximum resistance of the photoresistor, or its dark value. Thus, the $1.00 \mathrm{wt} . \%$ value exceeded the range of the SRS instrument.

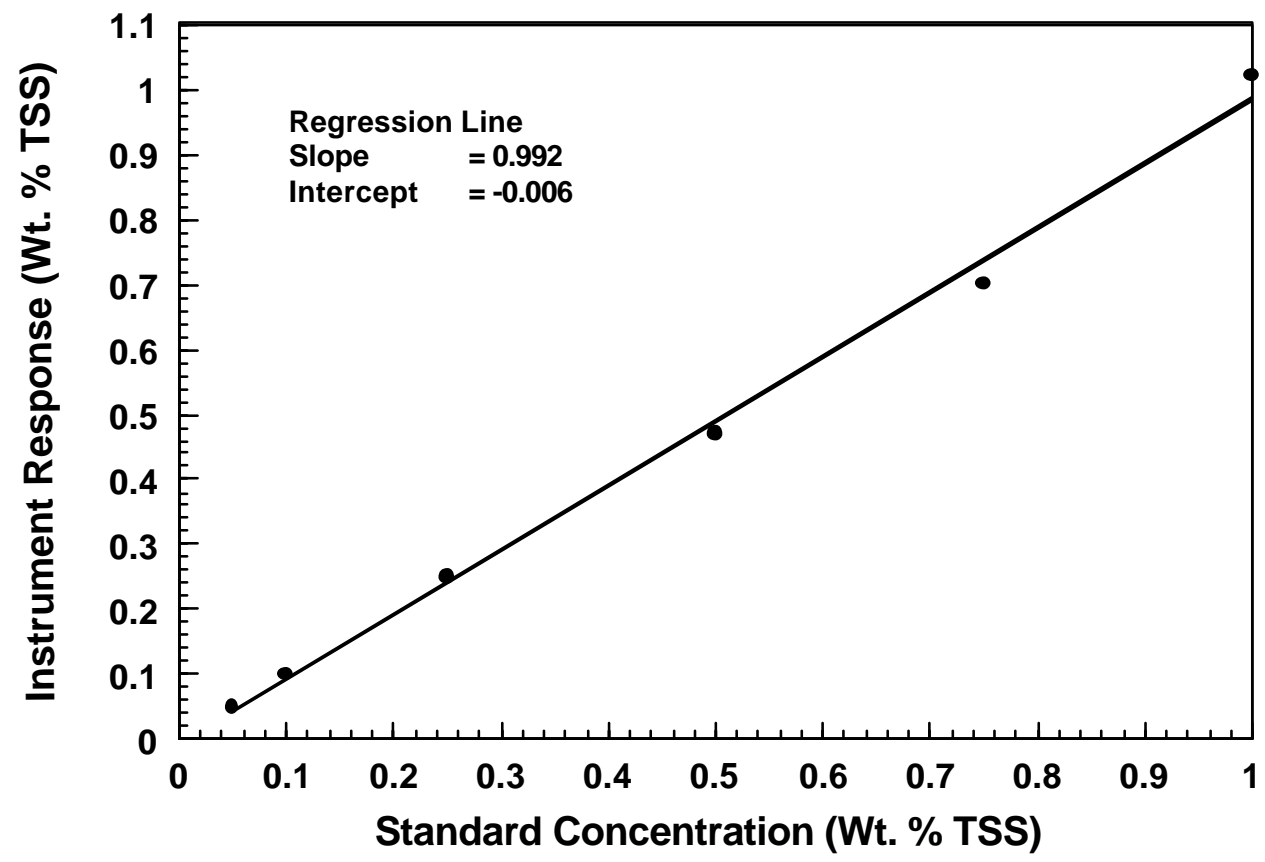

Figure 8. Plot showing the Monitek Instrument output versus the known wt.\% total suspended solids concentration for six calibration standards measured as unknowns. 


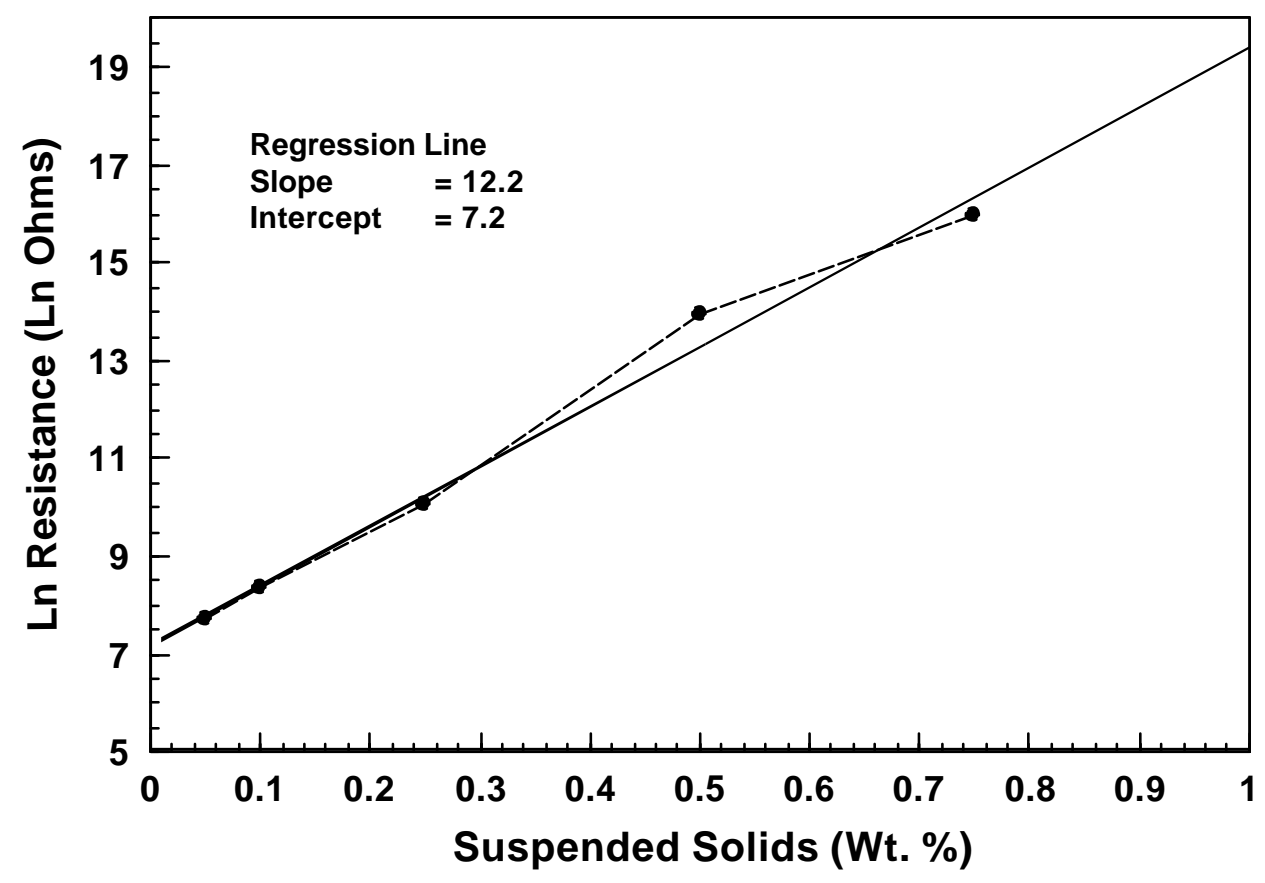

Figure 9. Plot showing the SRS sensor output versus the known wt. \% total suspended solids concentration for six calibration standards.

\subsection{Deployment Performance Characteristics}

To evaluate the deployment characteristics of the two sensors, a 5-inch diameter acrylic column was prepared to hold approximately 14 liters of a test suspension. The test suspension was prepared by diluting approximately 4 liters of the sludge surrogate provided by SRS to a total volume of 14 liters. The dilution was made with a solution of $\mathrm{NaOH}, \mathrm{NaNO}_{3}$, and $\mathrm{NaNO} 2$ in the concentrations of $0.12 \mathrm{M}, 0.25 \mathrm{M}, 0.15 \mathrm{M}$ respectively. Using this concentration of dissolved salts caused the sludge solids to flocculate, and allowed for rapid settling times, simulating the behavior of actual tank waste materials. The average settling rate for the supernatant/sludge interface was found to be approximately $0.2 \mathrm{~cm} / \mathrm{min}$ for the first few hours of settling, then slowing considerably. Using the previously determined wt\% solids concentration of the SRS waste surrogate, the concentration of total suspended solids in the column was determined to be approximately $5 \mathrm{wt}$. \% when it was thoroughly mixed. Fig ure 10 shows the column used for the tests. 


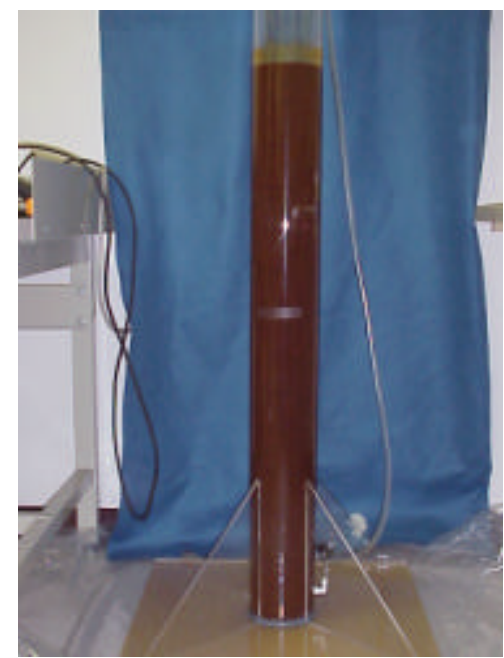

Figure 10. Photo of the test column with the sludge in a well-mixed state.

\subsubsection{Monitek Sensor}

Because of the configuration of the Monitek sensor, it is possible to determine very accurately the location of the supernatant/sludge interface with minimal disturbance of the interface itself. By lowering the probe through the supernatant column slowly, and only observing the instrument readout, it was possible to measure the position of the interface to within $1 \mathrm{~cm}$ of the true position. Figure 11 shows a sequence of photographs in which the Monitek sensor is lowered through the supernatant layer, into the sludge layer, and then withdrawn. The streamlined shape of the Monitek sensor, and the positioning of the optical path at the extreme lower end of the sensor housing, allow the sensor to create a minimal disturbance of the interface during the measurement process. 


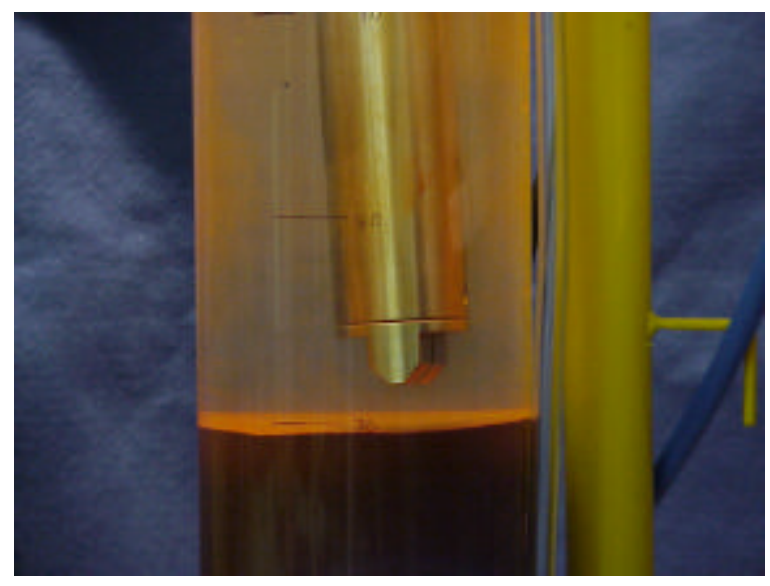

(a)

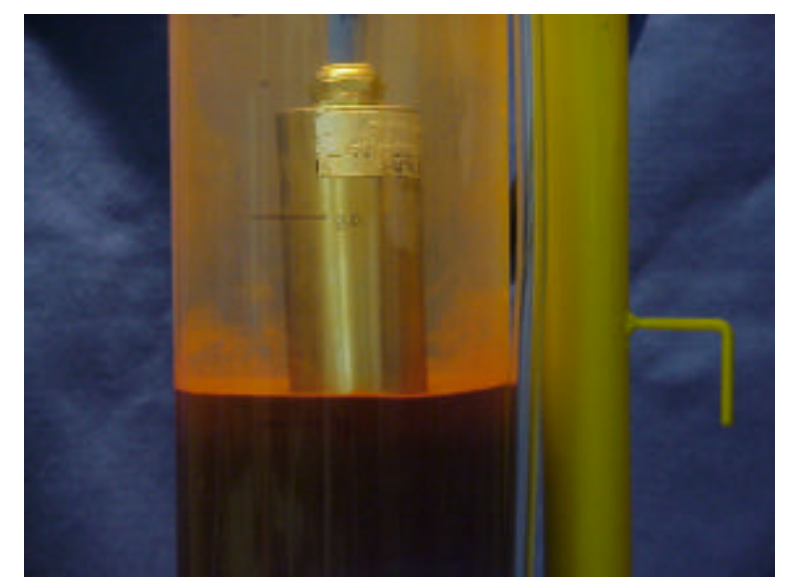

(c)

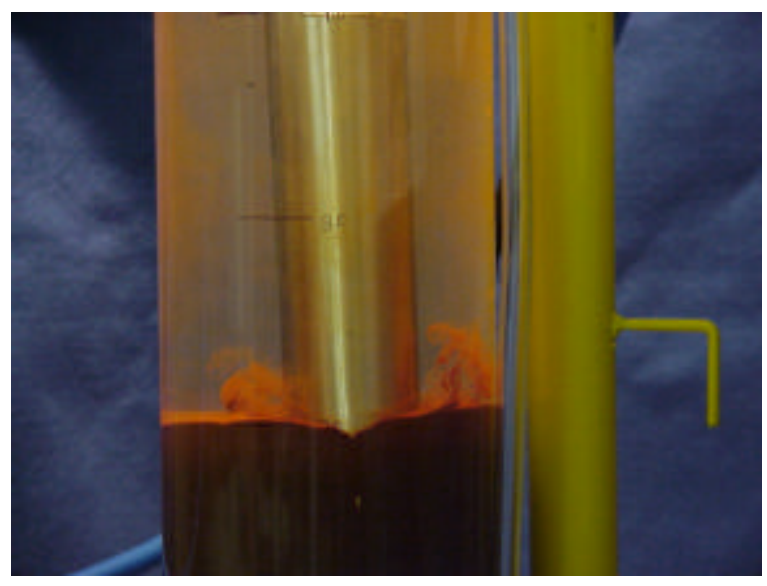

(b)

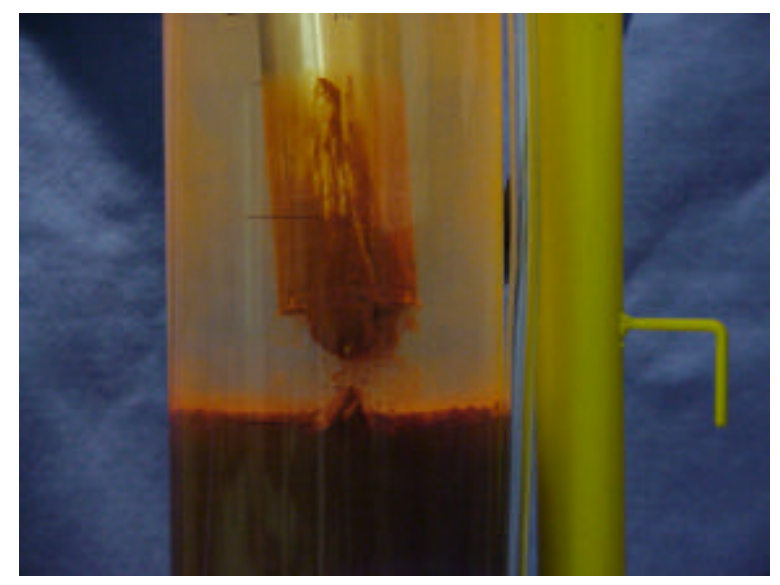

(d)

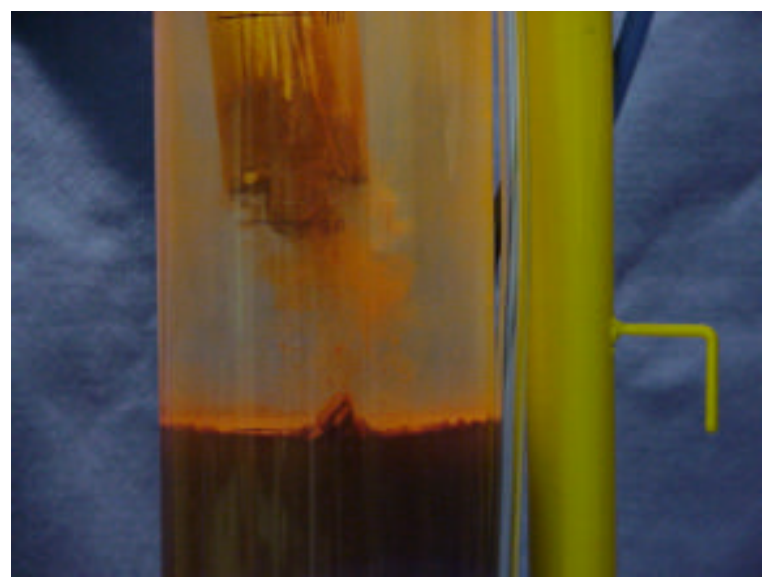

(e)

Figure 11 (a-e). Photo sequence showing the Monitek sensor being lowered through the supernatant liquid \& into the sludge layer, then being withdrawn. Note the relatively small disturbance of the supernatant/sludge interface as the sensor penetrates the interface in $8-b$, and the correspondingly small amount of sludge resuspended in 11-c. Photos 11-d \& 11-e show the sensor being withdrawn from the sludge layer and the relatively small amount of sludge that falls from the sensor to become resuspended in the supernatant layer. 


\subsubsection{SRS Sensor}

The SRS sensor also allows an accurate determination of the position of the supernatant/sludge interface. The SRS sensor, unlike the Monitek sensor however, causes a significant disturbance of the supernatant/sludge interface during the measurement process. This results from the fact that the position of the optical measurement is not at the extreme bottom of the sensor package. Rather it is approximately 6.5 " $(16.5 \mathrm{~cm})$ above the bottom of the sensor. This means that a substantial portion of the sensor must be deployed into the sludge before the sensor detects the presence of the supernatant/sludge interface.

Figures 12-a through 12-f show a sequence of photographs of the SRS sensor being deployed to the depth required to detect the interface, and then withdrawn. Note the significant disturbance of the interface as the steel weight passes through the interface, and the even larger disturbance of the interface as the sensor is withdrawn. The rather large volume of sludge adhering to the sensor is the result of the large surface area of those parts of the sensor that are submerged in the sludge, and the many nooks and crannies available to capture sludge. These include such features as the space between the metal tape and the power/signal cable, and the top surface of the steel weight. 


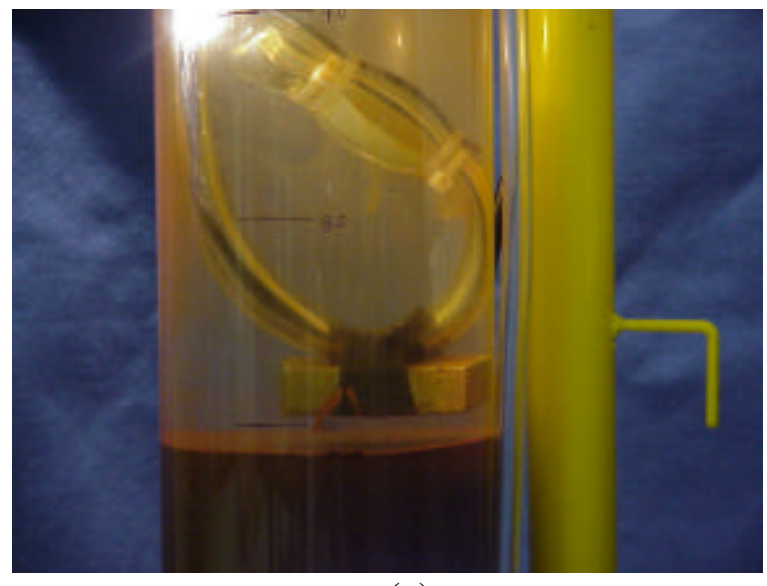

(a)

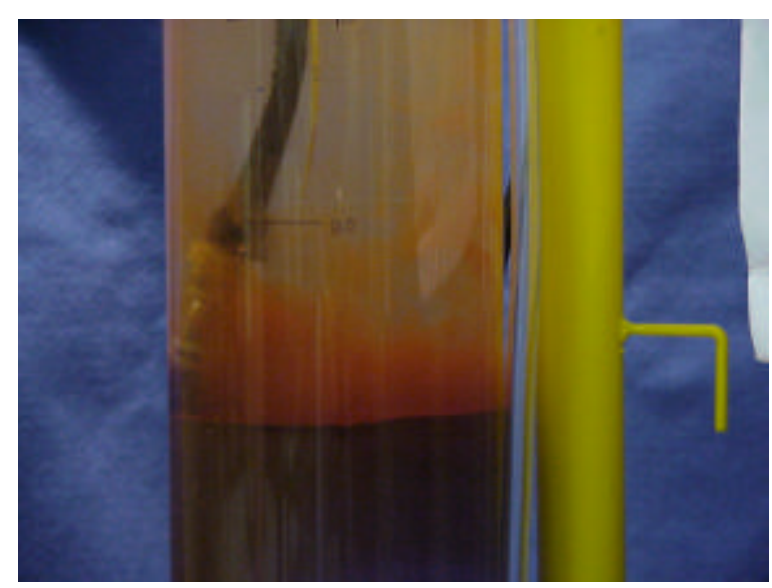

(c)

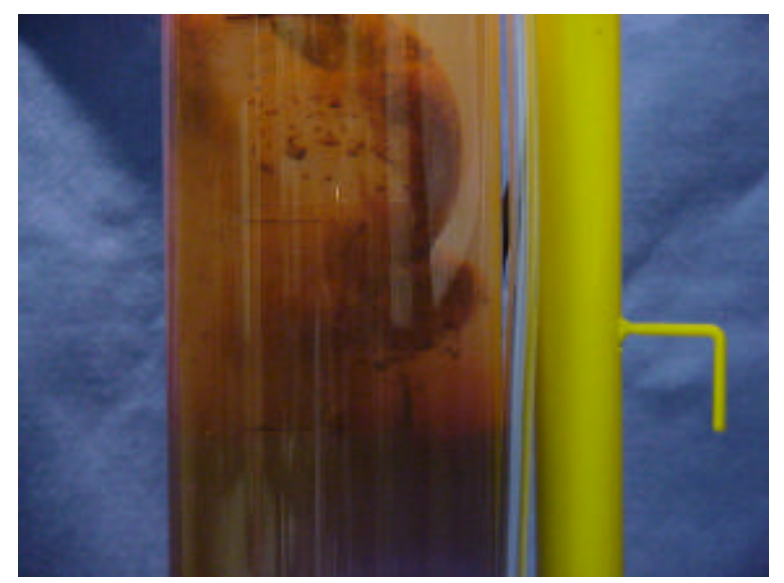

(e)

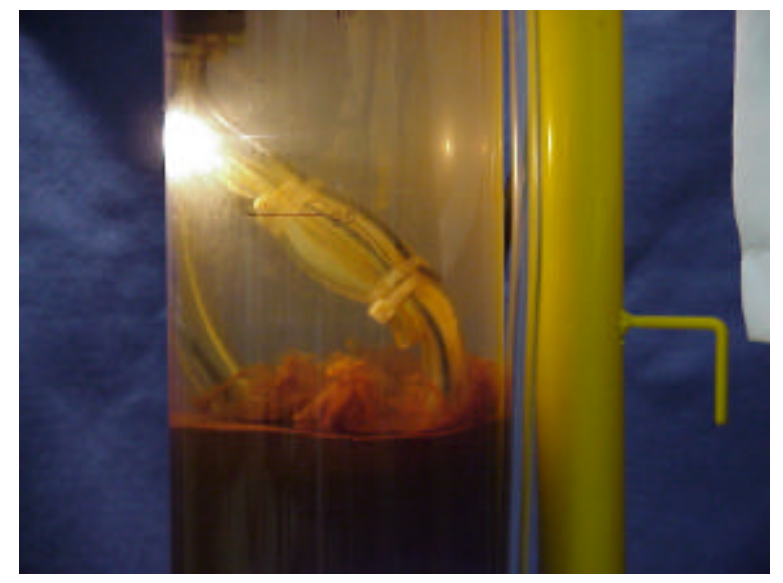

(b)

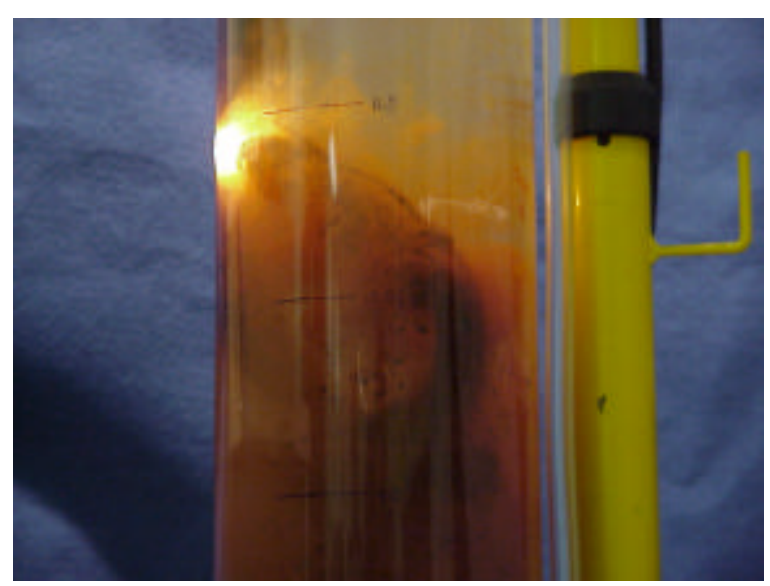

(d)

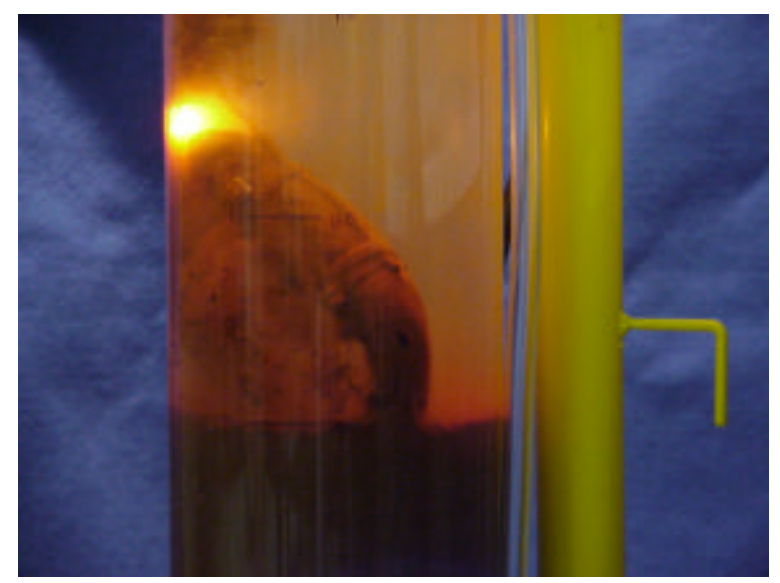

(f)

Figure 12 (a-f). Photo sequence showing the SRS sensor being lowered through the supernatant liquid $\&$ into the sludge layer, then being withdrawn. Note the relatively large disturbance of the supernatant/sludge interface as the sensor penetrates the interface in 12-b, and the correspondingly larger amount of sludge resuspended in 12-c than in 11-c. Photos 12-d through 12-f show the sensor being withdrawn from the sludge layer and the relatively large amount of sludge that is removed from the sensor from the sensor during retrieval to become resuspended in the supernatant layer. 


\subsection{Comparison of Sensor Performance}

To determine the effect of re-suspension of sludge in the supernatant layer by retrieval of the sensors upon subsequent measurements, a series of repetitive measurements were carried out with each sensor. The procedure followed was to lower the sensor into the test column slowly, only observing the instrument readout. As soon as the readout indicated that the interface was encountered, the depth was noted and the sensor was withdrawn approximately $20 \mathrm{~cm}$ and held for 1 minute. The output of the sensor at the end of one minute was noted as a measure of the amount of sludge re-suspension at this depth. Following the one minute interval, the sensor was again lowered until the interface was detected by observing the readout. The measurement cycle was repeated a total of ten times for each instrument. The column was allowed to settle overnight between the measurement series for the two instruments.

Table 1 shows the measurement results for each of the two instruments. The results are given as measured, i.e. in the depth units available from each device. The standard deviation for the SRS instrument is also given in $\mathrm{cm}$ to allow a ready comparison to the Monitek instrument. A trend of increasing depth to the interface is seen in the results for the Monitek instrument. This is most likely the result of some of the sludge adhering to the sensor housing as it is withdrawn, resulting in a slight depression in the surface of the sludge layer. The opposite trend is observed in the results for the SRS instrument, where the measured depth of the interface decreases with succeeding measurements. This results from disturbance of the sludge by the SRS sensor causing a mixing of the sludge and the supernatant liquid in the immediate vicinity of the interface. It should be noted, however, that in neither case are the measurement results of the interface depth significantly affected.

Table 1.

Replicate Measurements of Supernatant/Sludge Interface Depth

\begin{tabular}{|c|c|c|c|c|}
\hline \multirow[b]{2}{*}{ No. } & \multicolumn{2}{|c|}{ Monitek Instrument } & \multicolumn{2}{|c|}{ SRS Instrument } \\
\hline & $\begin{array}{l}\text { Measured Depth } \\
(\mathrm{cm})\end{array}$ & $\begin{array}{c}\text { Reading Between } \\
\text { Measurements (Wt. } \\
\%)^{*}\end{array}$ & $\begin{array}{l}\text { Measured Depth } \\
\text { (in.) }\end{array}$ & $\begin{array}{l}\text { Reading Between } \\
\text { Measurements } \\
\text { Ohms)* }\end{array}$ \\
\hline 1 & 101.5 & 0.06 & 44.5 & 4.4 \\
\hline 2 & 102.0 & 0.06 & 45.0 & 12.8 \\
\hline 3 & 102.5 & 0.05 & 44.0 & 4.6 \\
\hline 4 & 102.0 & 0.05 & 45.0 & 5.9 \\
\hline 5 & 102.0 & 0.05 & 44.5 & 2.7 \\
\hline 6 & 102.0 & 0.06 & 44.0 & 2.8 \\
\hline 7 & 102.5 & 0.08 & 44.5 & 2.6 \\
\hline 8 & 103.0 & 0.11 & 43.0 & 2.7 \\
\hline 9 & 103.0 & 0.06 & 43.0 & 2.6 \\
\hline 10 & 103.0 & 0.13 & 43.0 & 2.7 \\
\hline Mean & 102.35 & & 44.05 & \\
\hline Std. Dev. & 0.53 & & $0.8(2 \mathrm{~cm})$ & \\
\hline
\end{tabular}

* Measurement following a one minute wait while the sensor was held approximately $20 \mathrm{~cm}$ above the measured location of the interface. 


\subsection{Summary}

The chemistry conditions imposed on the suspended solids in these measurements caused the sludge to flocculate into relatively large flocs. This resulted in a rapid settling time, and a supernatant liquid that was very low in total suspended solids. When the column liquid was thoroughly mixed the total suspended solids (TSS) concentration was approximately $5 \mathrm{wt} \%$. As the solids were allowed to settle, the TSS concentration in the sludge layer became even higher. As a result of these conditions, the TSS concentration in the sludge layer always exceeded the upper limit of response for the SRS instrument, and exceeded the upper calibration limit for the Monitek instrument. The instruments could not, therefore, be used to measure a profile of the TSS concentration in the sludge layer, only to measure the depth of the interface. In this mode of operation, both instruments exhibited acceptable precision and accuracy, even under the conditions of repetitive measurement.

Visual observations demonstrate the benefits of the more streamlined shape of the Monitek instrument over the SRS instrument, and of the virtue of positioning the optics involved with the measurement at the lower most extreme of the sensor. Clearly, deployment of the Monitek sensor results in substantially less disturbance of the supernatant/sludge interface than is achievable with the SRS sensor. If the concentration of sludge is lower than in these tests, and there is a need to measure the TSS concentration profile of the sludge layer, the Monitek instrument will provide substantially better performance than the SRS instrument. In these measurements, where only the position of the interface was measured, the SRS sensor performed only slightly poorer than the Monitek instrument.

The calibration data show that the Monitek instrument will allow measurements at higher TSS concentrations than the SRS instrument. The Monitek instrument was able to obtain valid readings at a TSS concentration of $1 \mathrm{wt}$. \% of the SRS sludge surrogate, where as the SRS instrument could not. 


\subsection{RADIATION TESTING OF SENSORS}

Radiation survivability tests were completed as part of the SEAlevel project in order to assess the expected lifetime of a sensors system in the high radiation environment expected in DOE waste tanks. The tests were performed from September 21-23, 1998 at the Defense Microelectronics Activity (DMEA) System Engineering Gamma Irradiation Test (SEGIT) Facility located at McClellan Air Force Base, near Sacramento, CA.. The full test plan for the experimental effort is included as Appendix A.

In general, the results of the test were encouraging. Two sensors were exposed to well over 2.5 MR. One of the sensors is manufactured by Monitek and the other is manufactured by Zellweger. The response of each sensor to a nominal $0.1 \mathrm{wt}$. \% kaolinite solution was monitored while the sensors were being irradiated.

The sensor manufactured by Monitek survived quite well. The response of the sensor increased linearly with dose. However, this was due to color changes in the acrylic windows used with this model of the sensor. Therefore, there was essentially a continually increasing offset in the zero level of the sensor. The Monitek sensor that was to be used for SRS tank applications had sapphire windows, which are highly radiation tolerant. Therefore, there is little doubt that this sensor would survive the radiation environment found in DOE waste tanks. In addition, the cabling used with the sensor exhibited no signs of degradation due to the gamma rays.

The Zellweger instrument did not survive as well. At a cumulative dose of approximately 20,000 $\mathrm{R}$ the sensor output latched up (Fig. 13). A preliminary diagnosis of the failure mode indicated that a microswitch in the sensor failed. As such the Zellweger sensor was dropped from consideration.

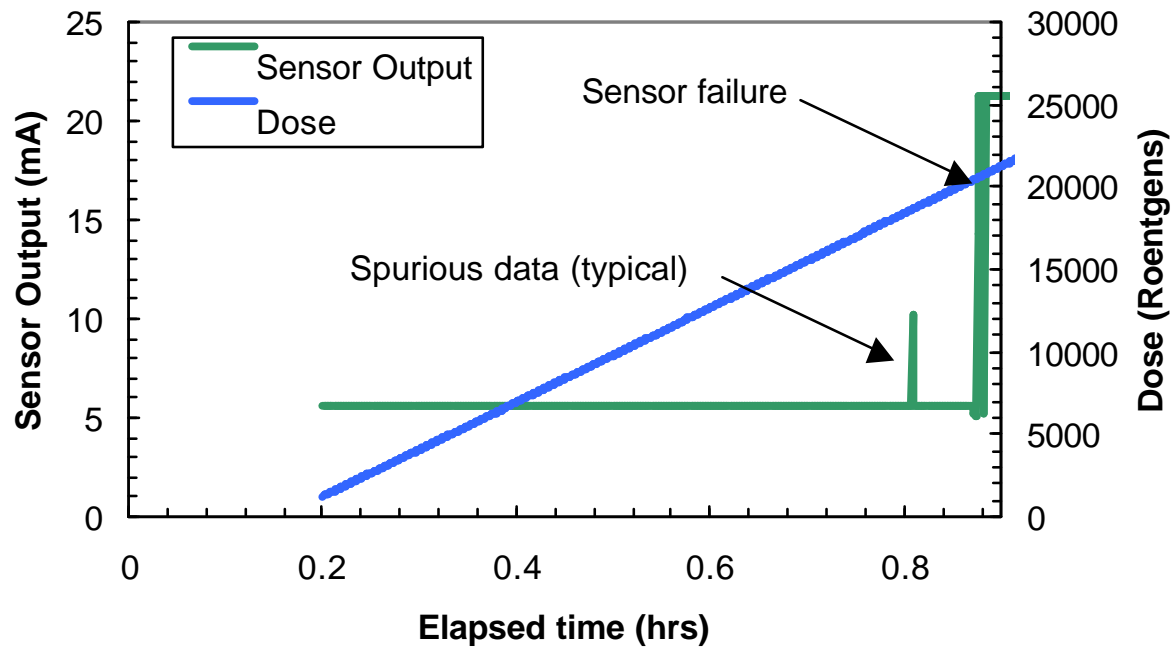

Figure 13. Results of radiation testing of the Zellweger instrument, showing sensor failure at 20,000 R. 


\title{
APPENDIX A - RADIATION TEST PLAN FOR TOTAL SUSPENDED SOLIDS INSTRUMENTS
}

\author{
August 1998
}

\section{David Cremer \\ Tom Kendrick}

Science \& Engineering Associates, Inc. 6100 Uptown Blvd., NE

Albuquerque, NM 87110

(505) $884-2300$ 


\section{A1 INTRODUCTION}

This test plan describes the test procedure and intended characterization tests for a total exposure radiation test to be conducted on two total suspended solids sensors, and one differential pressure transducer. The tests will be performed from September 21-23, 1998 at the Defense Microelectronics Activity (DMEA) System Engineering Gamma Irradiation Test (SEGIT) Facility located at McClellan Air Force Base, near Sacramento, CA.

\section{$1.1 \quad$ Intent}

The purpose of this test is to perform an evaluation of the viability of using LED type total suspended solids (TSS) sensors in a high level radioactive waste tank environment. This test is intended to deliver sufficient dose to the microelectronics contained within the TSS sensors so as to cause significant degradation in performance, or complete failure of the units. The TSS instruments will be operated during the radiation exposure, and the sensor output recorded on a data logger so that the manner in which the signal degrades prior to failure can be ascertained. Similarly the output from the differential pressure transducer will be recorded by the data logger, although it is not anticipated that this sensor will fail completely during the course of the exposure.

This test is not intended to provide insight into the specific degradation mechanisms of the components being tested.

\section{$1.2 \quad$ Background}

No data is available regarding the expected performance of these TSS probes in high radiation environments. The results of this test will be used in part to select between the two candidate TSS instrument systems, and in part to determine the design requirements for radiation shielding of a waste tank sensor incorporating a TSS probe.

\subsection{Summary}

Two waste tank sensors, manufactured by different vendors, will be tested in the radiation environment in the active mode. Both sensors will be exposed to a total exposure of $2.6 \mathrm{MR}$ at two discrete exposure rates of $500 \mathrm{R} /$ minute, and $1,500 \mathrm{R} /$ minute. The total exposure has been selected to provide potential qualification requirements, and the exposure rates have been selected to allow adequate time for the sensors to damage and for overall exposure uniformity. The tests will be conducted at ambient temperature.

\section{A2 REFERENCES AND DEFINITIONS}

\subsection{References}

The following references were used in the process of designing and conducting this test:

ANSI Standard N43.1, Radiation safety in design and operation of particle accelerators (also published as NIST Handbook 114).

ANSI Standard N433.1, Safe design and use of self-contained dry source storage gamma irradiators, category I (also published as NIST Handbook 127).

ANSI Standard N433.2, Panoramic dry-source storage irradiators, category II (to be published).

ASTM E666, Standard practice for calculating absorbed dose from gamma or x-radiation. 
ASTM E668-78, (Reapproved 1984) Standard practice for the application of thermoluminescence-dosimetry (TLD) systems for determining absorbed dose in radiationhardness testing of electronic devices.

EIA/TIA-455-A, Standard Test Procedure for Fiber Optic Fibers, Cables, Transducers, Sensors, Connecting and Terminating Devices, and Other Fiber Optic Components (AUG. 91)

\section{$2.2 \quad$ Test Conditions}

Testing of the TSS instruments and the differential pressure transducer for gamma radiation effects will include pre-irradiation characterization of the instrument performance as well as measurement of the instrument performance during the irradiation.

\subsubsection{Pre-irradiation Testing}

Prior to the irradiation tests the measurement repeatability of the TSS test instruments will be determined by repeated measurements of a series of kaolinite/iron oxide-in-water suspensions ranging from $0.05 \mathrm{wt}$. \% to $1.0 \mathrm{wt}$. \%. Additionally, the effect of temperature on the instrument response will be characterized by analyzing the kaolinite/iron oxide suspensions with the instrument probe and suspensions maintained at elevated temperatures ranging from $25{ }^{\circ} \mathrm{C}$ to $60{ }^{\circ} \mathrm{C}$. The measurement parameter for the pre-irradiation testing of the TSS instruments will be the uncalibrated output signal provided by the instruments' $4-20 \mathrm{~mA}$ output.

The differential pressure transducer/signal conditioner will undergo a similar characterization. The instrument will be calibrated against a reference pressure instrument (Dwyer Microtector). The zero and span controls will be adjusted to provide a zero volt output at zero differential pressure, and a 2.5 Volt output at $100 \%$ of the full scale value of the transducer. The measurement repeatability will be determined by repeatedly measuring a series of known differential pressures ranging from $10 \%$ to $100 \%$ of the rated full scale differential pressure. The instrument performance in elevated temperature environments will be determined by measuring a series of known differential pressures with the instrument equilibrated with elevated temperatures ranging from $25^{\circ} \mathrm{C}$ to $60^{\circ} \mathrm{C}$.

\subsubsection{In-Situ Testing}

The irradiation test will be an operationally active test. That is, the three sensors to be tested will be electrically and optically functioning during the irradiation. To accomplish this, the probes for the two suspended solids instruments will be immersed in a tank containing a kaolinite/iron oxidein-water suspension of know concentration. An air driven mixer will be used to ensure that the kaolinite/iron oxide suspension is well mixed for the duration of the irradiation. The probes will be connected to their respective signal conditioning/read-out modules and active measurements of the signal generated in response to the kaolinite/iron oxide suspension will be recorded during the irradiation via a data logger.

The differential pressure transducer will be positioned within the kaolinite/iron oxide suspension to ensure that it receives the same radiation exposure as the TSS instruments (within the exposure uncertainties in the chamber). Output from the signal conditioning/read-out module for the differential pressure transducer will be monitored during the irradiation via the data logger. Prior to the start of the irradiation, the calibration of the transducer will be verified, and corrected as necessary, using the Dwyer Microtector. During the irradiation, the differential pressure transducer will be connected to a constant source of fixed differential pressure.

The temperature of the kaolinite/iron oxide suspension will be monitored during the irradiation with two, type $\mathrm{K}$ thermocouples connected to the data logger. 


\section{A3 APPARATUS}

\subsection{Test Configuration}

The test apparatus will consist of the sensor test fixture, the gamma-ray exposure chamber, the in-situ measurement set-up, and the necessary calibration equipment. A block diagram of the measurement set-up is shown in Figure 1. The test configuration consists of two basic groupings of equipment. These are (1) equipment located within the test chamber that are intended to receive gamma dose during the irradiation, and (2) equipment that is located outside of the test chamber and is not intended to receive gamma dose during the irradiation. All three of the instruments to be tested, the two TSS instruments and one differential pressure instrument, are comprised of two functionally dependent components. These are (1) a sensor component that is exposed to the measurement environment, and (2) a signal conditioning/read-out module that is customarily located away from the measurement environment. For this test, only the sensor components will be exposed to the gamma radiation. The signal processing/readout electronics will be situated outside of the test chamber. This configuration of the instrumentation mimics the actual planned use of the equipment, where a sonde incorporating the TSS probe will be lowered into the high level radioactive waste tank and the signal conditioning/read-out modules will be located in a protected enclosure removed from the radiation fields of the tank waste.

A Campbell Scientific CR-10X data logger will be used to record the output signals from the two TSS instruments and the differential pressure transducer. The data logger will be programmed to sample the output signals at an interval not to exceed 20 seconds. Both TSS instruments are equipped with industry standard 4-20 mA current loop outputs, which will supply the necessary signal to the data logger through the use of a precision dropping resistor. The signal conditioning/read-out module used with the differential pressure transducer provides a user adjustable voltage output that will be connected directly to the data logger.

Other support equipment that will be situated outside of the test chamber include a compressor to power the air driven mixer, and a portable computer to down-load data, and interface with the CR-10X data logger.

The following table lists the various hoses and cables required to connect the sensors and equipment situated inside of the test chamber to equipment situated outside of the test chamber.

\begin{tabular}{|c|c|c|c|c|c|}
\hline No. & $\begin{array}{l}\text { I.D. } \\
\text { (inches) }\end{array}$ & $\begin{array}{c}\text { O.D. } \\
\text { (inches) }\end{array}$ & $\begin{array}{l}\text { Length } \\
\text { (m) }\end{array}$ & Description & Use \\
\hline 1 & $\mathrm{~N} / \mathrm{A}$ & 0.375 & 12 & Zellweger Sensor Cable & $\begin{array}{l}\text { Connect sensor to electronics } \\
\text { package }\end{array}$ \\
\hline 2 & $\mathrm{~N} / \mathrm{A}$ & 0.375 & & \begin{tabular}{|l|l} 
Monitek Sensor Cable \\
\end{tabular} & $\begin{array}{l}\text { Connect sensor to electronics } \\
\text { package }\end{array}$ \\
\hline 3 & $\mathrm{~N} / \mathrm{A}$ & 0.250 & & $\begin{array}{l}8 \text { Validyne Transducer } \\
\text { Cable }\end{array}$ & $\begin{array}{l}\text { Connect sensor to electronics } \\
\text { package }\end{array}$ \\
\hline 4 & $\mathrm{~N} / \mathrm{A}$ & 0.080 & & \begin{tabular}{l|l}
8 & Type K Thermocouple \\
\end{tabular} & Monitor Temperature of Sensors \\
\hline 5 & $\mathrm{~N} / \mathrm{A}$ & 0.080 & & 8 Type K Thermocouple & Monitor Temperature of Sensors \\
\hline 6 & 0.1875 & 0.406 & & $\begin{array}{l}8 \text { Braid-Reinforced PVC } \\
\text { Tubing }\end{array}$ & Air power line to Air Mixer \\
\hline 7 & 0.1875 & 0.406 & & $\begin{array}{l}8 \text { Braid-Reinforced PVC } \\
\text { Tubing }\end{array}$ & Air Power Return Line from Mixer \\
\hline 8 & 0.0625 & 0.125 & & 8 Vinyl Tubing & Pressure Transducer "+" \\
\hline 9 & 0.0625 & 0.125 & & 8 Vinyl Tubing & Pressure Transducer "_-" \\
\hline
\end{tabular}




\subsubsection{Environmental Test Conditions}

The test method will use the standard ambient test conditions specified in EIA/TIA-455-A. The tests will be performed at a nominal temperature of $25+/-5^{\circ} \mathrm{C}$. The temperature in the chamber is maintained by a nitrogen gas purge to the specified temperature to within $+/-5^{\circ} \mathrm{C}$. The temperature of the liquid bath will be monitored via type $\mathrm{K}$ thermocouples during the irradiation.

\subsection{Radiation Instrumentation}

\subsubsection{Steady State Gamma Measurements}

The test will use a cobalt-60 gamma radiation source operated in a steady state condition. The source will provide a uniform exposure rate across the face of the sensor test fixture to $+/-5 \%$. The average total exposure for the test is expressed in Roentgen $(R)$ to a precision of $+/-5 \%$ (steady state), traceable to national standards. The exposure rate shall be constant for at least $95 \%$ of the total dose exposure time of interest.

Caution: The energy of the gamma rays emitted by the source must be above $500 \mathrm{keV}$ to avoid serious complications with rapid variations in total dose as a function of depth within the test sample. This test configuration will include a filter box in accordance with ASTM Standard E1249-93.

The irradiation test facility for this experiment is capable of providing a steady state exposure via Co-60 gamma rays. The exposure rates to be used in this experiment are $500 \mathrm{R} /$ minute and $1,500 \mathrm{R} /$ minute. Co-60 provides a source of two gamma rays with energies of 1.17 and $1.3 \mathrm{MeV}$.

\subsubsection{Radiation Dosimetry}

The steady state gamma source exposure or exposure rate shall be measured prior to testing. The DMEA SEGIT facility will use a Model 9010 (S125) Radiation Monitor (S/N 90-1313) with a Model 90X5-0.18 (S125) lon Chamber (S/N 95-0478) for determining the exposure rate. Calibration of this dosimetry equipment is traceable to NIST. The coordinator of the SEGIT facility, along with a Science and Engineering Associates, Inc. representative, will perform exposure measurements at six (6) locations around the position of the test sample, with the probe located in the same plane as the sensors under test. An average of three (3) readings at each location will be obtained. This will be repeated for the two planned exposure rates of 500 $\mathrm{R} /$ minute and $1,500 \mathrm{R} /$ minute. Therefore a total of 36 readings will be taken. Following the irradiation the necessary calculations will be performed to convert the exposure to a dose in silicon, Rad (Si). 
Figure 1: Block Diagram of In-Situ Measurement Set-up

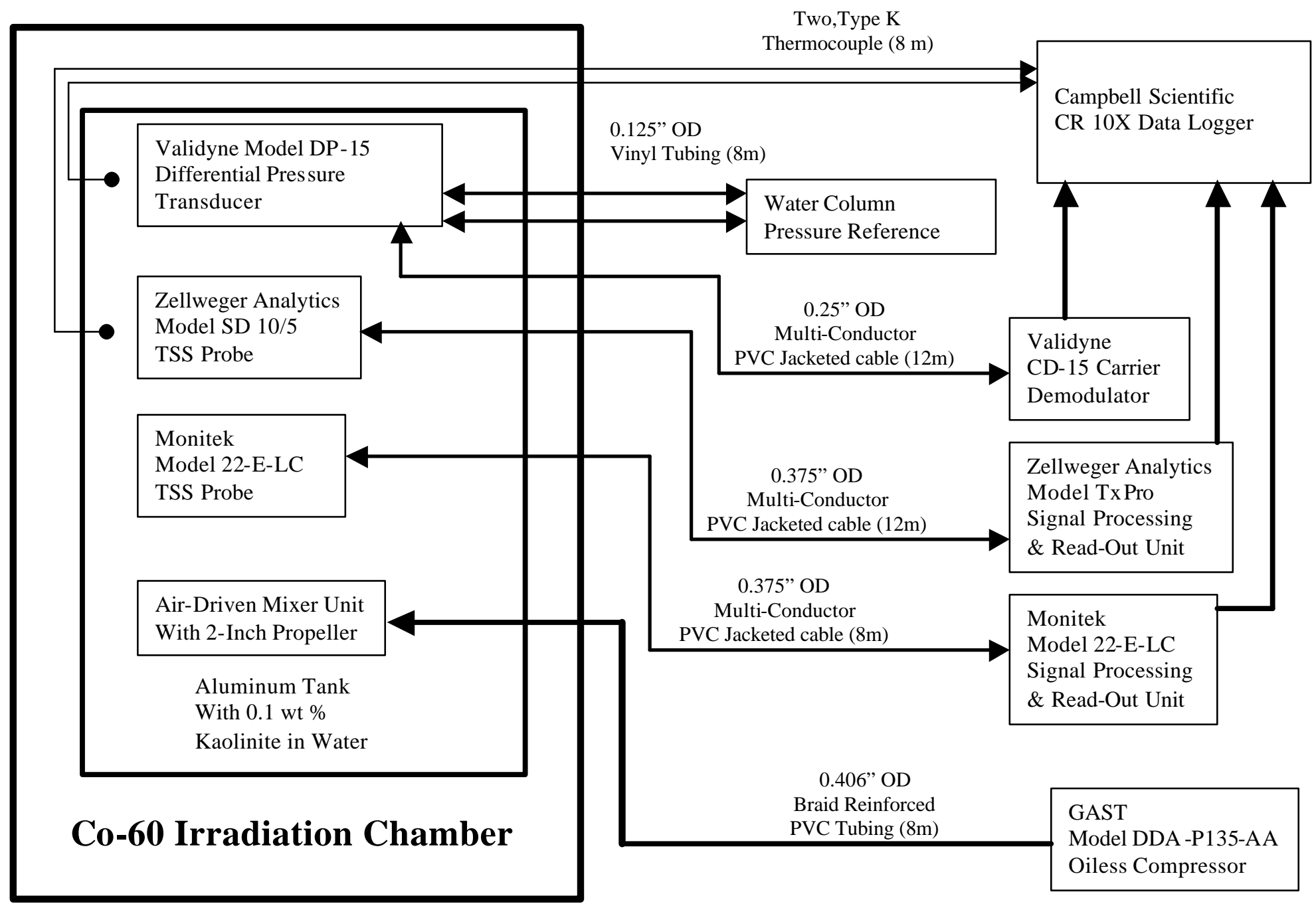




\subsection{Support Equipment}

Other support equipment that will be situated out side of the test chamber include a compressor to power the air driven mixer, and a portable computer to interface with, and down-load data from the CR-10X data logger. SEA will provide all equipment associated with operating and monitoring the instrumentation to be tested.

\subsection{Test Fixture}

The sensor test fixture will be an aluminum tank fabricated by SEA. The tank will be used to hold a 0.1 weight \% suspension of clay (kaolinite/iron oxide) in water. The kaolinite/iron oxide suspension is necessary to provide the probes with a suspended solids medium to measure during the irradiation. A sketch of the fixture showing the positioning of the test objects is shown in Figure 2. The air powered mixer is required to keep the kaolinite/iron oxide suspension well mixed during the irradiation.

\section{Figure 2. Sensor Test Fixture}

END VIEW

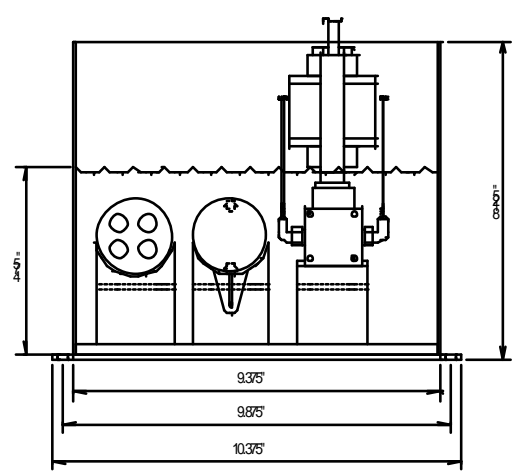

SIDE VIEW

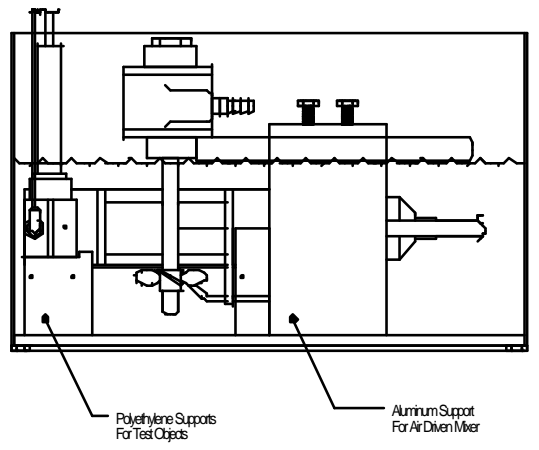

Box bottom is fabricated from 0.125 " aluminum.

Box sides are fabricated from 0.0625 " aluminum.

TOP VIEW

Hold down tabs are fabricated from 0.125 " aluminum and are thru-drilled for $1 / 4-20$ bolts.

Probes are positioned 0.25 " from front wall of tank.

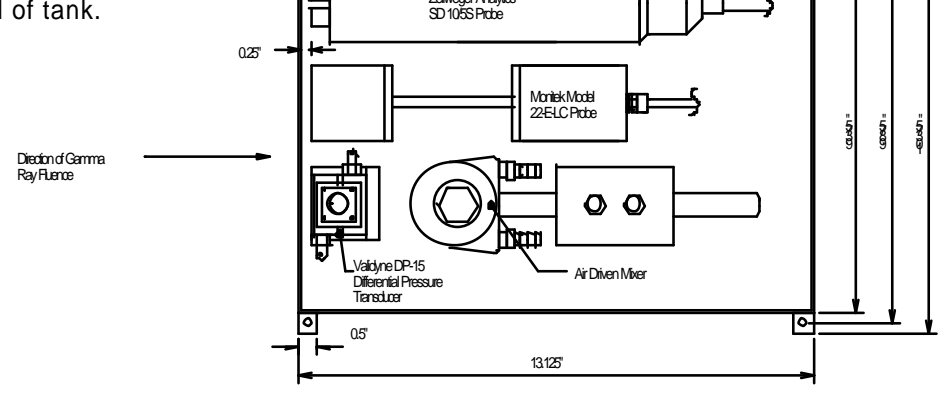

\subsection{Thermometers}

Two "Type K" thermocouple temperature sensors will be used for this test. Both thermocouples will be used to monitor the temperature of the kaolinite/iron oxide suspension during the irradiation. 


\section{A4 TEST SPECIMENS}

\subsection{Waste Tank Sensors}

Both TSS instruments have been pre-qualified to the extent that each has been shown to provide acceptable signal response over the expected range of suspended solids concentrations $(0.05 \mathrm{wt}$. \% to $1.00 \mathrm{wt}$ \%). For the intended application, the threshold suspended solids concentration is $0.1 \mathrm{wt} . \%$. Thus, the test will be executed with the instruments monitoring this concentration level.

Because the mode of failure of the various sensors is not known, determination of an unusable signal will be made by the SEA operator during the irradiation. Conditions that would cause the operator to declare that the signal is no longer useable include, but are not limited to: signal drops to 0 volts, signal raises to the equivalent of full scale output, signal exhibits random variations in level of a significant proportion of the original steady state value, signal exhibits excessive noise characteristics.

\section{A5 PROCEDURE}

\subsection{Pre-test}

\subsubsection{System Stability}

The stability of the total system will be verified under test conditions prior to subjecting the test instruments to gamma radiation exposure. A pretest stability check under operating conditions will be performed overnight to verify the stability of the sensors and the data logging system.

\subsubsection{Calibration of Radiation Source / Test Chamber}

The following procedure will be followed to determine the proper position of the test specimen in the chamber and ensure validity of the radiation exposure levels.

\subsubsection{System Calibration.}

The calibration certificate of the SEGIT Test Facility ionization chamber will be verified for currency.

\subsubsection{Exposure Profile and Uniformity.}

The cobalt 60 gamma radiation source will be checked for exposure profile uniformity and exposure rate. The exposure rate will be measured in at least six positions representative of the sensors location in the test fixture and the position determined to satisfy the exposure rate requirements in 3.2.1. In the event that the lower planned exposure rate $(500 \mathrm{R} / \mathrm{min})$ cannot be achieved, a contingency plan will be invoked. If the lowest achievable exposure rate is less than or equal to $700 \mathrm{R} / \mathrm{min}$, a two step exposure will still be used. In this case the lowest achievable exposure rate will be used, and the duration for this exposure rate will be set to deliver a total of $150 \mathrm{kR}$. The remainder of the $2.6 \mathrm{MR}$ will be delivered at 1,500 R/min for the appropriate duration. If the minimum practical exposure rate is greater than $700 \mathrm{R} / \mathrm{min}$, then the two step exposure will be abandon, and the entire exposure test will be executed at a single exposure rate of $1,400 \mathrm{R} / \mathrm{min}$. In the event that it is necessary to perform the test at a single exposure rate, the data acquisition rates will be modified to allow for a higher data collection density during the first part of the exposure, see Section 5.2.8. All options of the contingency plan will produce the same total exposure for the test.

\subsubsection{Thermocouple Calibration.}

The calibration of the thermocouples in the test chamber will be checked.

\subsubsection{Differential Pressure Instrument Calibration}


The calibration parameters of the differential pressure instrument will be verified, and adjusted as necessary, using the Dwyer Microtector pressure reference.

\subsection{Sensor Test}

\subsubsection{Positioning of the Sensors Under Test}

The sensor test fixture shown in Figure 2 will be attached to a laboratory jack, which in turn will be attached to a plywood sheet. With this setup the front to back adjustment and side to side adjustment of the sensors can be accomplished by moving the plywood sheet. Once correct positioning of the sensors is achieved, the plywood will be secured to the test chamber to prevent movement. Vertical adjustment of the sensor position will be accomplished with the laboratory jack. Once the proper positioning of the jack has been achieved, it will be locked into place.

The sensor test fixture will be detachable from the laboratory jack through four screws. In this manner the fixture can be removed and put back into the chamber with confidence that the sensor position in the irradiation chamber is constant.

\subsubsection{Dosimetry}

To conduct the dosimetry measurements, the sensors will be removed from the sensor test fixture. The ionization chamber will be placed in the positions where the sensors will be. All exposure determinations will be relative to the front face of the sensors. The fixture will be placed onto the laboratory jack and the exposure will be measured. After completion of the exposure measurements the dosimeter will be removed from the sensor test fixture and the sensors will be reinstalled.

\subsubsection{Sensor Connections}

The TSS sensors will be connected to their respective signal conditioning/read-out modules and the output signals from the modules will be connected to the data logger. The output cable from the signal conditioning/read-out module for the pressure transducer will be connected to the data logger. The thermocouple sensors will also be connected to the data logger.

\subsubsection{Total Suspended Solids Solution}

The sensor test fixture will be filled with a known solution of 0.1 wt.\% kaolinite/iron oxide in water. The air driven mixer will be connected to the Gast compressor via a length of PVC tubing. The exhaust port of the mixer will be connected to a similar length of tubing that is routed out of the test chamber and terminates in a muffler. The compressor will then be turned on, and the air flow rate adjusted to provide sufficient mixing of the suspension.

\subsubsection{Stability Tests}

The data logger program will be initiated and the output of all the sensors will be analyzed to verify proper sensor/data acquisition performance. The system will be left running overnight so that sensor baselines and stability can be established.

\subsubsection{Test Readiness}

The data obtained from the overnight stability test will be analyzed to confirm that the test apparatus and data collection system are operating correctly. System functionality will be verified prior to gamma irradiation.

\subsubsection{Test Initiation}


The operator will ensure that the data logger is collecting data. Irradiation of the test objects will be initiated and the test commencement time will be noted in the test notebook. The time at which the fullscale exposure rate is achieved $(1,500 \mathrm{R} / \mathrm{min})$ will also be noted in the notebook.

\subsubsection{Data acquisition}

Data from five instruments will be recorded during the tests; TSS sensor \#1, TSS sensor \#2, pressure transducer, thermocouple \#1, and thermocouple \#2. All data will be recorded with a Campbell scientific data logger in increments of 20 seconds per data point (maximum) for the planned two step exposure rate test. In the event that the test must be run at a single exposure rate of $1,400 \mathrm{R} / \mathrm{min}$, the data collection density will be adjusted as follows: For the first 75 minutes of the test, data will be acquired in $5 \mathrm{~s}$ increments for a resulting dose resolution of approximately $116 \mathrm{R} /$ data point. After 75 minutes, the total dose on the test objects will be approximately $105 \mathrm{R}$. At this time the data acquisition rate will be reduced to once every 20 seconds resulting in an exposure resolution of approximately $467 \mathrm{R} /$ data point. Irradiation of the test objects will continue for a total elapsed time of 31 hours resulting in a total dose of 2.6 MR. In the event that all test components are irradiated to failure prior to the full 31 hours the test will be terminated.

\subsubsection{Exposure Rates}

The initial exposure rate for the tests will be $500 \mathrm{R} /$ minute. This exposure rate will continue for a period of five hours, resulting in a net exposure of $150 \mathrm{kR}$. The exposure rate will then be increased to 1,500 $\mathrm{R} /$ minute and will continue for a period of 27 hours. Thus, at the end of the 32 hour test period the net exposure to the sensors will be $2.58 \mathrm{MR}$. The time at which the exposure rate is changed will be noted in the test notebook.

\subsubsection{Test Termination}

Irradiation of the test objects will continue for a total elapsed time of 32 hours resulting in a total exposure of $2.58 \mathrm{MR}$. In the event that all test components are irradiated to failure prior to the full 32 hours, the test will be terminated.

The time at which the exposure begins to be reduced and the time at which all irradiation ceases will be noted in the laboratory notebook. Data from the sensors will continue to be recorded for a 20-minute period after irradiation is terminated. Immediately afterwards, the data from the data logger will be downloaded to a PC. A backup disk of the data will also be made. 


\section{APPENDIX B - DESIGN DRAWINGS OF THE SEALEVEL SYSTEM}

Due to the size of the drawings (ANSI D size), the SEAlevel design drawings are provided separately. 